Supporting Information

\title{
1,2-Thiaphosphetanes: The quest for Wittig-type ring cleavage, rearrangement and sulfur atom transfer
}

\section{Arturo Espinosa Ferao, ${ }^{\text {a) }}$ Rainer Streubel ${ }^{\text {b) }}$}

a Depto. Química Orgánica, Facultad de Química, Campus de Espinardo, Universidad de Murcia, 30100 Murcia (Spain), E-mail: artuesp@um.es.

${ }^{\mathrm{b}}$ Institut für Anorganische Chemie der Rheinischen Friedrich-Wilhelms-Universität Bonn, GerhardDomagk-Straße 1, 53121 Bonn (Germany), E-mail: r.streubel@uni-bonn.de.

\section{Table of contents}

Figure S 1: Computed relative energy profile for P-S bond elongation in 8a. S 2

$\begin{array}{lr}\text { Figure S 2: Computed } \mathrm{TSP}^{\mathrm{H}} \text {. } & \text { S } 2\end{array}$

Calculated structures: Cartesian coordinates and energies for all minima and transition states. S 3 


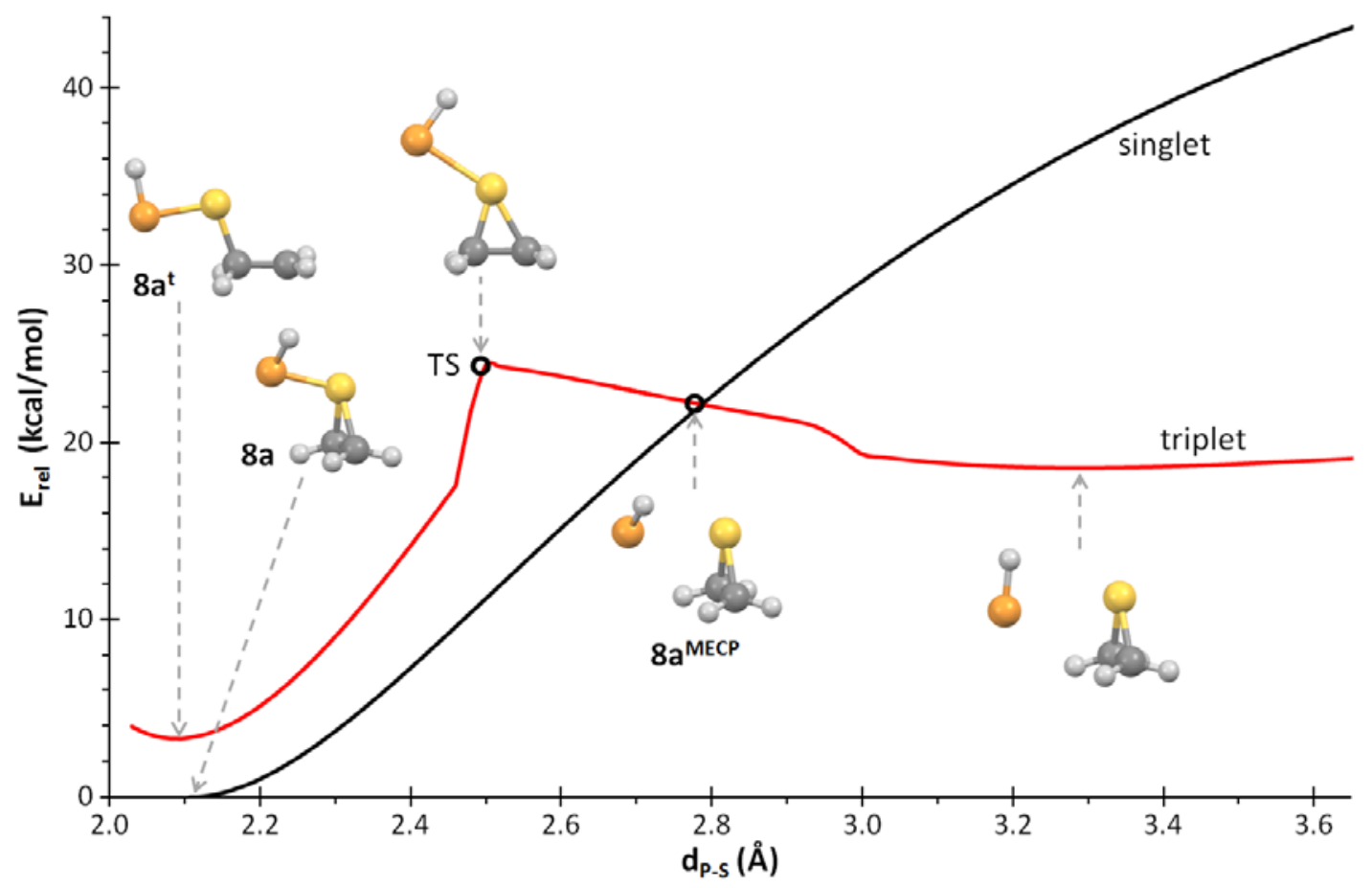

Figure S1. Computed (B3LYP-D3/def2-TZVP) relative (electronic) energy profile for the P-S bond elongation scan in 8a.

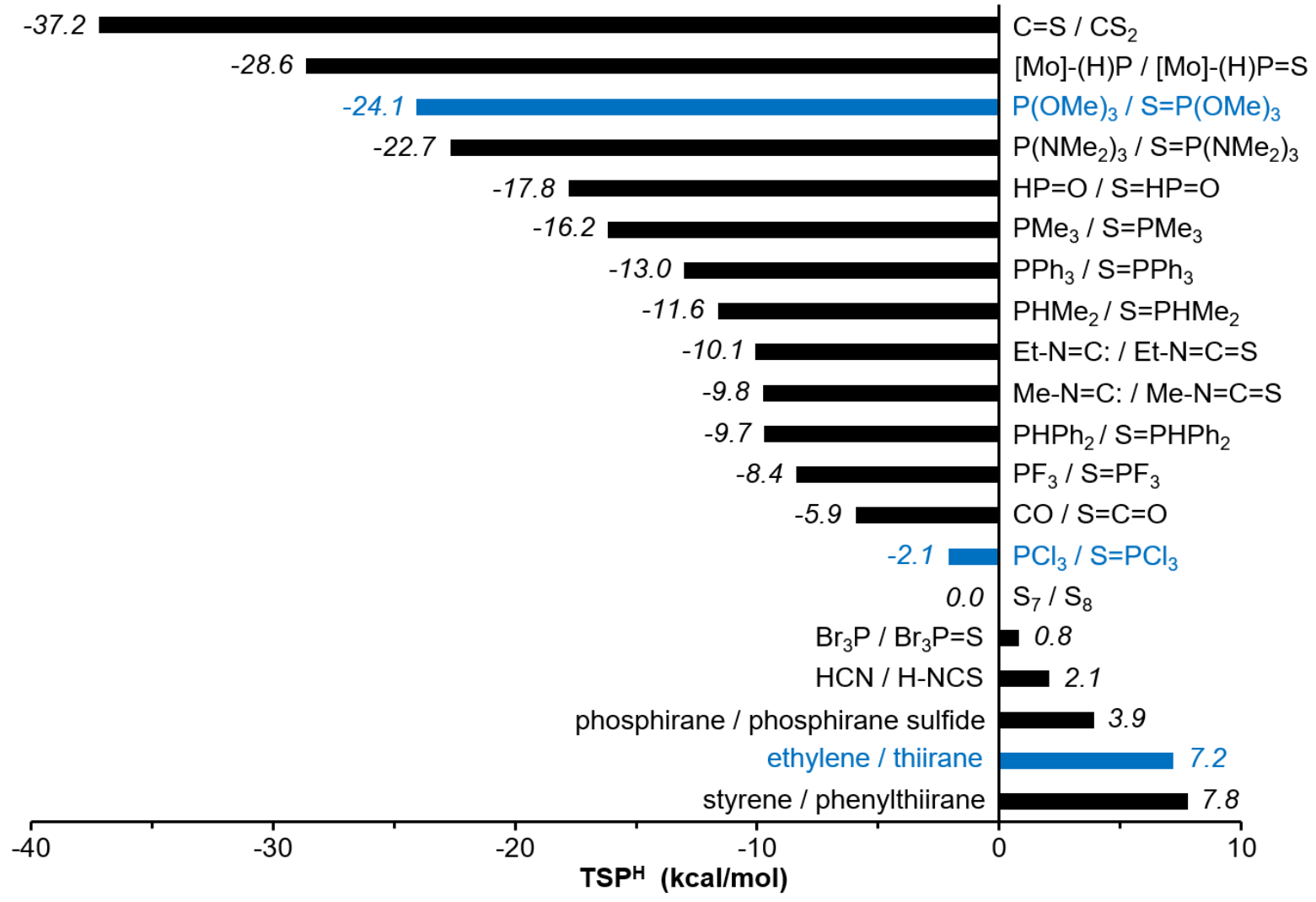

Figure S2. Computed (DLPNO-CCSD(T)/def2-TZVPP(еср)//B3LYP-D3/def2-TZVP(ecp)) enthalphybased thermodynamic S-transfer potentials $\left(\mathrm{TPS}^{\mathrm{H}}\right)$ according to equation 1. [Mo] stands for Mo(CO) 5 . 


\section{Calculated structures}

Cartesian coordinates (in $\AA$ ), G correction (G-E) and ZPE (in hartrees) for minima and transition states were computed at B3LYP-D3/def2-TZVP(ecp). Electronic energies (in hartrees) are quoted at the (gasphase) DLPNO-CCSD(T)/def2-TZVPP level unless otherwise stated. Imaginary frequencies are obtained upon frequency calculation (at the optimization level).

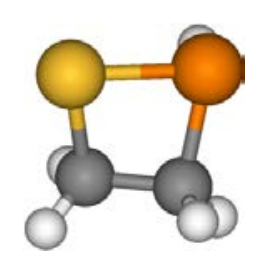

1a:

$$
\operatorname{TS}\left(\mathbf{1 a}^{\text {P-inv}}\right) \text { : }
$$

P $\quad-0.694944$

$\mathrm{H} \quad-0.924225$

S $\quad 0.886740$

C -1.488392

H $\quad-2.255659$

$$
\begin{aligned}
& E=-817.69880979382 \mathrm{au} \\
& \mathrm{ZPE}=0.06635142 \mathrm{au} \\
& \mathrm{G}_{\text {corr }}=0.03938544 \mathrm{au}
\end{aligned}
$$

$\begin{array}{lrrr}\mathrm{P} & -0.825343 & 0.147701 & -0.729040 \\ \mathrm{H} & -0.962738 & 1.311067 & 0.088956 \\ \mathrm{~S} & 1.039157 & -0.555946 & 0.121771 \\ \mathrm{C} & -1.440368 & -1.045447 & 0.586152 \\ \mathrm{H} & -2.349519 & -0.744403 & 1.107351\end{array}$

$\begin{array}{lrrr}\mathrm{H} & -1.606677 & -2.012516 & 0.110545 \\ \mathrm{C} & -0.165607 & -1.060024 & 1.436938 \\ \mathrm{H} & 0.094402 & -2.018144 & 1.886464 \\ \mathrm{H} & -0.189866 & -0.291757 & 2.210687\end{array}$

$$
\begin{aligned}
& E=-817.616427994735 \mathrm{au} \\
& \mathrm{ZPE}=0.06502537 \mathrm{au} \\
& \mathrm{G}_{\mathrm{corr}}=0.03792770 \mathrm{au} \\
& v=-1052.02 \mathrm{~cm}^{-1}
\end{aligned}
$$

$$
\operatorname{TS}(\mathbf{1 a} \rightarrow \mathbf{2 a} \mathbf{a}):
$$

$$
\begin{aligned}
& E=-817.586063527921 \mathrm{au} \\
& Z P E=0.06031672 \mathrm{au} \\
& G_{\text {corr }}=0.03309250 \mathrm{au} \\
& v=-344.24 \mathrm{~cm}^{-1}
\end{aligned}
$$

$\begin{array}{lrrr}\mathrm{H} & -1.868671 & -1.826899 & 0.236037 \\ \mathrm{C} & -0.148704 & -1.209766 & 1.485627 \\ \mathrm{H} & 0.352265 & -2.084141 & 1.076458 \\ \mathrm{H} & -0.264971 & -1.302269 & 2.563591\end{array}$

$\begin{array}{lrrr}\mathrm{P} & -0.936454 & 0.295652 & -0.462438 \\ \mathrm{H} & -0.636348 & 1.164975 & 0.650635 \\ \mathrm{~S} & 0.719670 & -0.997046 & -0.048050 \\ \mathrm{C} & -1.998298 & -0.925374 & 0.266649 \\ \mathrm{H} & -3.074040 & -0.814089 & 0.150273\end{array}$

$\begin{array}{lrrr}\mathrm{H} & -1.644627 & -1.936601 & 0.369436 \\ \mathrm{C} & 0.379496 & -1.051365 & 1.601773 \\ \mathrm{H} & 0.921692 & -1.767626 & 2.208438 \\ \mathrm{H} & -0.137652 & -0.237997 & 2.083109\end{array}$




$$
E=-380.698210963334 \mathrm{au}
$$$$
\mathrm{ZPE}=0.03350323 \mathrm{au}
$$$$
\mathrm{G}_{\text {corr }}=0.01107915 \mathrm{au}
$$

$\begin{array}{lrrrrrrr}\mathrm{P} & -1.971882 & 1.099872 & 0.259239 & \mathrm{H} & -1.369008 & -0.923144 & -0.857926 \\ \mathrm{H} & -2.631103 & 1.075141 & 1.524737 & \mathrm{H} & -2.196118 & -1.294897 & 0.735080 \\ \mathrm{C} & -1.842051 & -0.542930 & 0.041079 & & & & \end{array}$

3:

$$
\begin{aligned}
& E=-436.929943542961 \mathrm{au} \\
& Z P E=0.02464303 \mathrm{au} \\
& G_{\text {corr }}=0.00271185 \mathrm{au}
\end{aligned}
$$

$\begin{array}{lrrr}\mathrm{P} & -1.971882 & 1.099872 & 0.259239 \\ \mathrm{H} & -2.631103 & 1.075141 & 1.524737 \\ \mathrm{C} & -1.842051 & -0.542930 & 0.041079\end{array}$

$\mathrm{TS}(\mathbf{1 a} \rightarrow \mathbf{4 + 5 a}):$

4:

$$
\begin{aligned}
& E=-78.439324272117 \mathrm{au} \\
& \mathrm{ZPE}=0.05091781 \mathrm{au} \\
& \mathrm{G}_{\text {corr }}=0.02978132 \mathrm{au} \\
& \mathrm{H}_{\text {corr }}=0.05490083 \mathrm{au}
\end{aligned}
$$

$\begin{array}{llllllll}\mathrm{C} & -1.846400 & -2.156783 & 1.508935 & \mathrm{C} & -1.238354 & -0.994229 & 1.693590 \\ \mathrm{H} & -1.348470 & -3.098321 & 1.708332 & \mathrm{H} & -0.217863 & -0.935398 & 2.053144 \\ \mathrm{H} & -2.866890 & -2.215547 & 1.149366 & \mathrm{H} & -1.736343 & -0.052722 & 1.494187\end{array}$

\section{5a:}

$$
\mathrm{E}=-739.217079473346 \mathrm{au}
$$

\begin{tabular}{|c|c|c|c|c|c|c|}
\hline & & $\begin{array}{l}\mathrm{ZP} \\
\mathrm{G}_{\mathrm{Cc}}\end{array}$ & & & & \\
\hline 1.911873 & 1.711411 & -0.383367 & $\mathrm{~S}$ & 0.296519 & -0.192193 & -1.249610 \\
\hline 0.646599 & 1.701096 & -1.075882 & & & & \\
\hline
\end{tabular}$$
\mathrm{ZPE}=0.00869885 \mathrm{au}
$$$$
\mathrm{G}_{\text {corr }}=-0.01448014 \mathrm{au}
$$
H $\quad-1.369008$
$-0.923144 \quad-0.857926$
$-1.294897$
0.735080

$\begin{array}{lrrrrrrr}\mathrm{P} & -0.588344 & -0.008992 & -0.526668 & \mathrm{H} & -1.805363 & -2.144444 & 0.266579 \\ \mathrm{H} & -0.896954 & 1.129942 & 0.284226 & \mathrm{C} & -0.433708 & -1.049039 & 1.499657 \\ \mathrm{~S} & 1.433865 & -0.338535 & -0.188101 & \mathrm{H} & 0.283460 & -1.846805 & 1.567322 \\ \mathrm{C} & -1.607803 & -1.187387 & 0.732909 & \mathrm{H} & -0.315434 & -0.231265 & 2.195475\end{array}$


1b:

$\begin{array}{lrrr}\text { Mo } & 0.074149 & -0.040324 & 2.554041 \\ \text { C } & 0.092835 & 0.056619 & 4.588200 \\ \text { O } & 0.102713 & 0.104373 & 5.730032 \\ \text { C } & 1.983558 & 0.722484 & 2.446297 \\ \text { O } & 3.042388 & 1.140159 & 2.358410 \\ \text { C } & -0.703587 & 1.870278 & 2.440102 \\ \text { O } & -1.137757 & 2.921729 & 2.362294 \\ \text { C } & -1.831818 & -0.808196 & 2.554899 \\ \text { O } & -2.887814 & -1.243260 & 2.525846 \\ \text { C } & 0.839179 & -1.946124 & 2.640423\end{array}$

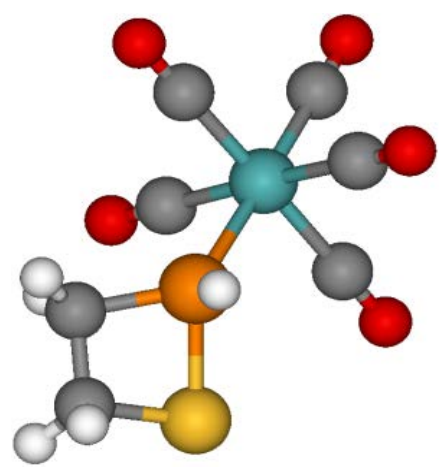

$\mathrm{E}=-1451.46993871172 \mathrm{au}$

$\mathrm{ZPE}=0.10918616 \mathrm{au}$

$\mathrm{G}_{\text {corr }}=0.06212816 \mathrm{au}$
$\mathrm{TS}(\mathbf{1 b} \rightarrow \mathbf{2 b}+\mathbf{3})$ :

$$
\begin{aligned}
& E=-1451.35573573841 \mathrm{au} \\
& Z P E=0.10366273 \mathrm{au} \\
& \mathrm{G}_{\mathrm{corr}}=0.05657499 \mathrm{au} \\
& v=-376.16 \mathrm{~cm}^{-1}
\end{aligned}
$$

$\begin{array}{lrrr}\mathrm{O} & 1.261056 & -3.007150 & 2.684772 \\ \mathrm{P} & 0.034705 & -0.207451 & 0.095108 \\ \mathrm{H} & 1.270650 & -0.140004 & -0.595314 \\ \mathrm{~S} & -1.201702 & 0.992501 & -1.173997 \\ \mathrm{C} & -1.234175 & -0.642672 & -2.054811 \\ \mathrm{H} & -2.223411 & -0.858277 & -2.453366 \\ \mathrm{H} & -0.509430 & -0.631213 & -2.867736 \\ \mathrm{C} & -0.789130 & -1.543937 & -0.892047 \\ \mathrm{H} & -0.155746 & -2.388299 & -1.162390 \\ \mathrm{H} & -1.644673 & -1.905750 & -0.32257\end{array}$

$\begin{array}{lrrr}\text { Mo } & 0.063341 & -0.119715 & 2.576023 \\ \text { C } & 0.347151 & 0.187767 & 4.569508 \\ \text { O } & 0.510738 & 0.367182 & 5.686323 \\ \text { C } & 1.852827 & 0.791923 & 2.138698 \\ \text { O } & 2.845907 & 1.287336 & 1.868480 \\ \text { C } & -0.922483 & 1.685005 & 2.431635 \\ \text { O } & -1.479800 & 2.677146 & 2.340041 \\ \text { C } & -1.728605 & -1.069061 & 2.931017 \\ \text { O } & -2.715615 & -1.609502 & 3.120700 \\ \text { C } & 1.009502 & -1.944425 & 2.697896\end{array}$

O 1.520961

P $\quad-0.235653$

$\mathrm{H} \quad 0.938971$

S $\quad-1.428327$

C $\quad-0.865719$

$\mathrm{H} \quad-1.465376$

H 0.160561

C $\quad-1.076555$

$\mathrm{H} \quad-1.000877$

H $\quad-1.948961$

$\begin{array}{rr}-2.963409 & 2.764391 \\ -0.499234 & 0.156396 \\ -0.293975 & -0.624649 \\ 0.791932 & -1.134375 \\ -0.021881 & -2.483592 \\ -0.016563 & -3.384940 \\ -0.354921 & -2.543025 \\ -1.879658 & -0.521198 \\ -2.828914 & 0.000718 \\ -1.741545 & -1.131862\end{array}$

2b:

$\begin{array}{lr}\text { Mo } & 0.005566 \\ \text { C } & 0.322613 \\ \text { O } & 0.506032 \\ \text { C } & 1.299606 \\ \text { O } & 2.020778 \\ \text { C } & -1.575502\end{array}$

$\begin{array}{rr}-0.423678 & 2.479063 \\ 0.602625 & 4.201991 \\ 1.187543 & 5.166375 \\ 0.875006 & 1.529335 \\ 1.604231 & 1.030999 \\ 0.800622 & 1.990626\end{array}$

$$
\begin{aligned}
& E=-1014.46135462208 \mathrm{au} \\
& Z P E=0.07654829 \mathrm{au} \\
& G_{\text {corr }}=0.03249052 \mathrm{au}
\end{aligned}
$$

$\begin{array}{lrrr}\mathrm{O} & -2.452623 & 1.473114 & 1.705699 \\ \mathrm{C} & -1.285054 & -1.695324 & 3.472753 \\ \mathrm{O} & -1.995650 & -2.393487 & 4.027230 \\ \mathrm{C} & 1.585966 & -1.672440 & 2.905009 \\ \mathrm{O} & 2.464395 & -2.365595 & 3.129414 \\ \mathrm{P} & -0.386615 & -1.670023 & 0.390947\end{array}$




$\begin{array}{lrrrrrrr}\mathrm{H} & 0.443157 & -2.713345 & -0.077155 & \mathrm{H} & -2.363523 & -0.870525 & -0.678031 \\ \mathrm{C} & -1.562760 & -1.593461 & -0.764618 & \mathrm{H} & -1.577914 & -2.245023 & -1.627279\end{array}$

\begin{tabular}{|c|c|c|c|c|c|c|}
\hline \multicolumn{2}{|c|}{$\mathrm{TS}(\mathbf{1 b} \rightarrow \mathbf{4}+\mathbf{5 b})$} & $\begin{array}{l}\mathrm{E}= \\
\mathrm{ZP} \\
\mathrm{G}_{\mathrm{C}} \\
v=\end{array}$ & $4 \mathrm{a}$ & & & \\
\hline Mo -0.006879 & -0.040758 & 2.455736 & $\mathrm{O}$ & 1.188400 & -2.993864 & 2.244122 \\
\hline 0.142511 & -0.001029 & 4.496795 & $\mathrm{P}$ & -0.076034 & 0.080182 & 0.009483 \\
\hline 0.235742 & 0.025071 & 5.634352 & $\mathrm{H}$ & 1.134962 & -0.233528 & -0.654491 \\
\hline 1.901754 & 0.723115 & 2.300690 & $\mathrm{~S}$ & -0.964769 & 1.524617 & -1.074378 \\
\hline 2.958253 & 1.140227 & 2.196609 & $\mathrm{C}$ & -1.053831 & -1.026933 & -1.978608 \\
\hline-0.802555 & 1.876381 & 2.491299 & $\mathrm{H}$ & -2.019937 & -0.655843 & -2.277201 \\
\hline-1.244043 & 2.923679 & 2.526255 & $\mathrm{H}$ & -0.255230 & -0.931600 & -2.701803 \\
\hline-1.922325 & -0.797028 & 2.525973 & $\mathrm{C}$ & -0.863057 & -1.698276 & -0.769673 \\
\hline-2.981989 & -1.221891 & 2.526835 & $\mathrm{H}$ & -0.016546 & -2.364644 & -0.661237 \\
\hline 0.764986 & -1.936693 & 2.348836 & $\mathrm{H}$ & -1.737424 & -1.945698 & -0.181410 \\
\hline
\end{tabular}

5b:

$\mathrm{E}=-1372.98986999961 \mathrm{au}$

$\mathrm{ZPE}=0.05222159 \mathrm{au}$

$\mathrm{G}_{\text {corr }}=0.00783457 \mathrm{au}$

$\begin{array}{lrrr}\mathrm{P} & 0.548823 & 0.981127 & 0.078932 \\ \mathrm{H} & 1.876998 & 1.270938 & -0.342498 \\ \mathrm{~S} & -0.600988 & 1.297432 & -1.431824 \\ \text { Mo } & 0.171249 & 0.293859 & 2.345663 \\ \mathrm{C} & -0.124836 & -0.247508 & 4.313067 \\ \mathrm{O} & -0.283452 & -0.538836 & 5.402808 \\ \mathrm{C} & 2.107660 & 0.849688 & 2.748796\end{array}$

O 3.190487

1.155207

2.935836

C $\quad-0.534138$

2.188533

2.813715

O $\quad-0.930529$

3.222512

3.074285

C $\quad-1.745569$

$-0.256251$

1.794834

O $\quad-2.790457$

$-0.559990$

1.464143

C $\quad 0.819764 \quad-1.634473$

1.944143

C $\quad 2.107660$

O 1.168155

$-2.697422$

1.734149

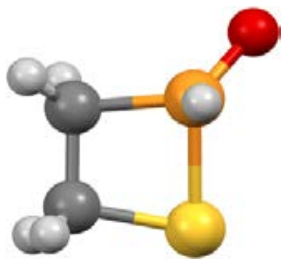

1c:

$E=-892.869420440428 \mathrm{au}$

$\mathrm{ZPE}=0.07130226 \mathrm{au}$

$\mathrm{G}_{\text {corr }}=0.04276545 \mathrm{au}$

$\begin{array}{lrrr}\mathrm{P} & 0.003390 & -0.007647 & 0.000049 \\ \mathrm{H} & -0.023121 & 0.023858 & 1.413644 \\ \mathrm{~S} & 2.065854 & 0.013786 & -0.484409 \\ \mathrm{C} & 0.155667 & -1.772196 & -0.440373 \\ \mathrm{H} & -0.371201 & -2.478953 & 0.200135\end{array}$

$\begin{array}{rrrr}\mathrm{H} & -0.187910 & -1.888711 & -1.468522 \\ \mathrm{C} & 1.696766 & -1.806060 & -0.356669 \\ \mathrm{H} & 2.177080 & -2.349210 & -1.166562 \\ \mathrm{H} & 2.054151 & -2.187273 & 0.597043 \\ \mathrm{O} & -0.973311 & 0.900777 & -0.622480\end{array}$




\begin{tabular}{|c|c|c|c|c|c|c|c|}
\hline \multicolumn{3}{|c|}{$\mathrm{TS}(\mathbf{1 c} \rightarrow \mathbf{2 c}+\mathbf{3})$} & \multicolumn{3}{|c|}{$\begin{array}{l}E=-892.754710932409 \mathrm{au} \\
Z P E=0.06587267 \mathrm{au} \\
\mathrm{G}_{\mathrm{corr}}=0.03714754 \mathrm{au} \\
v=-255.79 \mathrm{~cm}^{-1}\end{array}$} & & \\
\hline & -0.084667 & -0.233651 & 0.086474 & $\mathrm{H}$ & 0.057195 & -2.272791 & -1.274737 \\
\hline 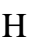 & 0.623626 & -0.321579 & 1.303681 & $\mathrm{C}$ & 2.307241 & -1.580802 & -0.302666 \\
\hline S & 1.800924 & -0.273963 & -1.173285 & $\mathrm{H}$ & 2.850136 & -2.381013 & -0.789337 \\
\hline C & -0.537703 & -1.760593 & -0.542911 & $\mathrm{H}$ & 2.189229 & -1.628447 & 0.772876 \\
\hline & -1.594045 & -2.006533 & -0.504103 & $\mathrm{O}$ & -1.014573 & 0.907745 & 0.095866 \\
\hline
\end{tabular}

2c:

$E=-455.8445201462 \mathrm{au}$

$\mathrm{ZPE}=0.03794745 \mathrm{au}$

$\mathrm{G}_{\text {corr }}=0.01352733 \mathrm{au}$

$\begin{array}{lrrr}\mathrm{P} & -0.011759 & -0.023694 & -0.023127 \\ \mathrm{H} & -0.047976 & 0.004648 & 1.379446 \\ \mathrm{C} & 1.526443 & 0.007500 & -0.552555\end{array}$

$\begin{array}{llll}\mathrm{H} & 1.794987 & -0.007237 & -1.597737\end{array}$

$\begin{array}{llll}\mathrm{H} & 2.303395 & 0.045903 & 0.198673\end{array}$

$\begin{array}{llll}\mathrm{O} & -1.312708 & -0.074094 & -0.71106\end{array}$

$\operatorname{TS}(\mathbf{1 c} \rightarrow \mathbf{4}+\mathbf{5 c}):$

$$
\begin{aligned}
& E=-892.802568282298 \mathrm{au} \\
& \mathrm{ZPE}=0.06862300 \mathrm{au} \\
& \mathrm{G}_{\mathrm{corr}}=0.03977121 \mathrm{au} \\
& v=-418.87 \mathrm{~cm}^{-1}
\end{aligned}
$$

$\begin{array}{lrrr}\mathrm{P} & -0.000494 & -0.000580 & -0.000019 \\ \mathrm{H} & 0.001277 & 0.002281 & 1.409789 \\ \mathrm{~S} & 1.856096 & 0.000476 & -0.646230 \\ \mathrm{C} & -0.571969 & -1.999195 & -0.068367 \\ \mathrm{H} & -1.246176 & -2.042203 & 0.778328\end{array}$

$\begin{array}{rrrr}\mathrm{H} & -1.031478 & -2.115076 & -1.042651 \\ \mathrm{C} & 0.755177 & -2.387437 & 0.093786 \\ \mathrm{H} & 1.366359 & -2.691506 & -0.740192 \\ \mathrm{H} & 1.205071 & -2.469482 & 1.074312 \\ \mathrm{O} & -1.111549 & 0.770869 & -0.581662\end{array}$

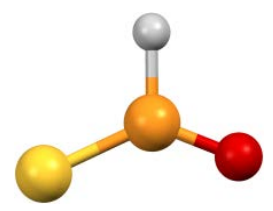

\section{5c:}

$$
\begin{aligned}
& E=-814.389043434222 \mathrm{au} \\
& \mathrm{ZPE}=0.01443187 \mathrm{au} \\
& \mathrm{G}_{\text {corr }}=-0.01071886 \mathrm{au} \\
& \mathrm{H}_{\text {corr }}=0.01891331 \mathrm{au}
\end{aligned}
$$

$\begin{array}{lrrrrrrr}\mathrm{H} & -0.034943 & 0.000122 & 1.399784 & \mathrm{~S} & 1.726462 & 0.000116 & -0.756579 \\ \mathrm{P} & -0.008087 & -0.000462 & -0.008208 & \mathrm{O} & -1.361427 & -0.001426 & -0.573077\end{array}$


1d:

$\mathrm{TS}(\mathbf{1 d} \rightarrow \mathbf{2 d}+\mathbf{3}):$

2d:

$\mathrm{TS}(\mathbf{1 d} \rightarrow \mathbf{4}+\mathbf{5 d}):$

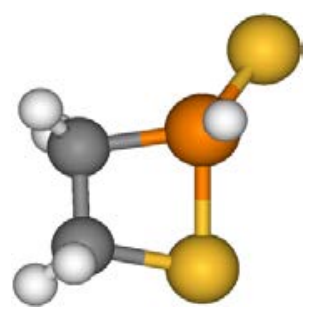

$E=-1215.4624099031 \mathrm{au}$

$\mathrm{ZPE}=0.06936634 \mathrm{au}$

$\mathrm{G}_{\text {corr }}=0.03976758 \mathrm{au}$

$\begin{array}{lrrr}\mathrm{P} & -0.119519 & -0.068736 & 0.057007 \\ \mathrm{H} & 1.233580 & 0.197993 & -0.244472 \\ \mathrm{~S} & -1.013468 & 1.869708 & 0.046672 \\ \mathrm{C} & -0.930002 & -0.160079 & -1.588054 \\ \mathrm{H} & -0.343148 & -0.630009 & -2.376615\end{array}$

H $\quad-1.866326$

C $\quad-1.167173$

H $\quad-2.146571$

H $\quad-0.396518$

S $\quad-0.366865$
$-0.702319$

1.355918

1.619591

1.843285

$-1.369000$
$-1.459894$

$-1.732757$

$-2.124695$

$-2.325985$

1.449769

$\begin{array}{lrrr}\mathrm{P} & -0.112993 & -0.174111 & -0.147004 \\ \mathrm{H} & 0.904017 & 0.738800 & -0.494598 \\ \mathrm{~S} & -1.688333 & 1.475624 & -0.087500 \\ \mathrm{C} & -0.890934 & -0.831634 & -1.538128 \\ \mathrm{H} & -0.681805 & -1.871031 & -1.768041\end{array}$

$$
-0.147004
$$

$$
\begin{aligned}
& E=-1215.34732358656 \mathrm{au} \\
& \mathrm{ZPE}=0.06384281 \mathrm{au} \\
& \mathrm{G}_{\mathrm{corr}}=0.03398977 \mathrm{au} \\
& v=-294.03 \mathrm{~cm}^{-1}
\end{aligned}
$$$$
\text { H } \quad-1.822378
$$$$
\text { C } \quad-1.181516
$$

\begin{tabular}{|c|c|c|c|c|c|c|c|}
\hline \multicolumn{8}{|c|}{$\begin{array}{l}\mathrm{ZPE}=0.03625903 \mathrm{au} \\
\mathrm{G}_{\mathrm{corr}}=0.01065223 \mathrm{au}\end{array}$} \\
\hline $\mathrm{P}$ & -0.006402 & -0.002116 & -0.024461 & $\mathrm{H}$ & -1.581814 & 1.795796 & -0.007108 \\
\hline $\mathrm{H}$ & 1.397345 & 0.002647 & 0.007219 & $\mathrm{H}$ & 0.200227 & 2.345546 & 0.048190 \\
\hline $\mathrm{C}$ & -0.530622 & 1.550004 & 0.008777 & $\mathrm{~S}$ & -0.920490 & -1.684624 & -0.09462 \\
\hline
\end{tabular}$$
\text { H }-1.900645
$$

\begin{tabular}{|c|c|c|c|c|c|c|c|}
\hline \multicolumn{3}{|c|}{$\operatorname{TS}(\mathbf{1 d} \rightarrow \mathbf{4}+\mathbf{5 d})$} & & $4 \mathrm{a}$ & & & \\
\hline $\mathrm{P}$ & 0.488800 & -0.064982 & -0.021700 & $\mathrm{H}$ & -1.771492 & -0.901541 & -1.898978 \\
\hline $\mathrm{H}$ & 1.343257 & 0.282067 & 1.075586 & $\mathrm{C}$ & -1.318731 & 1.246252 & -1.598450 \\
\hline S & -0.036624 & 1.973994 & -0.500183 & $\mathrm{H}$ & -2.284804 & 1.314696 & -1.103685 \\
\hline $\mathrm{C}$ & -0.969750 & -0.181699 & -1.859144 & $\mathrm{H}$ & -1.335596 & 1.812219 & -2.535635 \\
\hline $\mathrm{H}$ & -0.146009 & -0.385730 & -2.529044 & $\mathrm{~S}$ & -1.085061 & -1.138923 & 0.672212 \\
\hline
\end{tabular}$$
\text { H } \quad-0.129922
$$$$
\text { S } 0.388499
$$

$\begin{array}{rr}-0.432143 & -1.892810 \\ 1.960028 & -1.584745 \\ 2.318083 & -2.310738 \\ 2.038501 & -1.831280 \\ -1.265767 & 1.355822\end{array}$




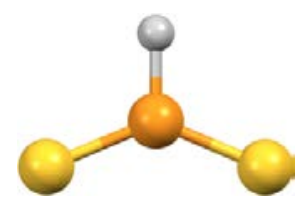

$$
\text { 5d: } \quad \begin{aligned}
& \mathrm{E}=-1136.9857693387 \mathrm{au} \\
& \mathrm{ZPE}=0.01260142 \mathrm{au} \\
& \mathrm{G}_{\text {corr }}=-0.01372617 \mathrm{au}
\end{aligned}
$$$$
\begin{array}{lllr}
\mathrm{P} & 0.012348 & 0.376301 & 0.421764 \\
\mathrm{H} & 0.978569 & 0.536467 & -0.590203
\end{array}
$$

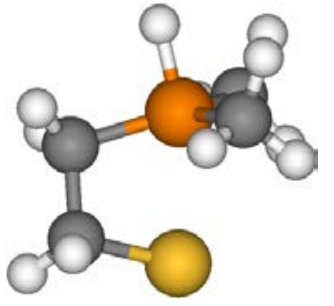

1e:

$$
\mathrm{E}=-897.366459956461 \mathrm{au}
$$

$\mathrm{ZPE}=0.14226087142 \mathrm{au}$

$\mathrm{G}_{\text {corr }}=0.10993898 \mathrm{au}$

S $\quad-0.923913 \quad 1.989286 \quad 0.815436$

S $\quad-0.069692 \quad-1.406210 \quad 1.091919$

$\begin{array}{rrrrrrrr}\mathrm{P} & 0.076630 & -0.044791 & 0.016988 & \mathrm{C} & -0.071671 & -0.917209 & -1.574764 \\ \mathrm{~S} & 2.519187 & -0.024540 & -0.070318 & \mathrm{H} & -1.103080 & -1.221555 & -1.757212 \\ \mathrm{C} & 0.588561 & 1.723918 & -0.002851 & \mathrm{H} & 0.254934 & -0.243922 & -2.367222 \\ \mathrm{H} & -0.117247 & 2.330433 & -0.580522 & \mathrm{H} & 0.597257 & -1.774220 & -1.572797 \\ \mathrm{H} & 0.535576 & 2.054003 & 1.038314 & \mathrm{C} & 0.079215 & -1.161531 & 1.458471 \\ \mathrm{C} & 2.030524 & 1.677465 & -0.504948 & \mathrm{H} & -0.900121 & -1.628238 & 1.578228 \\ \mathrm{H} & 2.666552 & 2.419870 & -0.023195 & \mathrm{H} & 0.864301 & -1.905308 & 1.340831 \\ \mathrm{H} & 2.077175 & 1.840905 & -1.582989 & \mathrm{H} & 0.307430 & -0.567971 & 2.344155 \\ \mathrm{H} & -1.330628 & 0.272974 & 0.146442 & & & & \end{array}$

\section{$1 \mathrm{e}^{\mathrm{H}-\mathrm{eq}:}$}

$\begin{array}{lr}\mathrm{P} & 0.010384 \\ \mathrm{~S} & 2.123879 \\ \mathrm{C} & 0.684386 \\ \mathrm{H} & -0.115497 \\ \mathrm{H} & 1.215543 \\ \mathrm{C} & 1.658875 \\ \mathrm{H} & 2.521075 \\ \mathrm{H} & 1.169014 \\ \mathrm{H} & 0.541110\end{array}$

$\operatorname{TS}(\mathbf{1 e} \rightarrow \mathbf{2 e}+\mathbf{3}):$

$$
\begin{aligned}
& E=-897.359868696429 \mathrm{au} \\
& \mathrm{ZPE}=0.14201477 \mathrm{au} \\
& \mathrm{G}_{\text {corr }}=0.11009250 \mathrm{au}
\end{aligned}
$$
$0.057946 \quad 0.073045$
$0.275919-1.190429$
$1.737829 \quad 0.418290$
$2.473340 \quad 0.555083$
$1.639931 \quad 1.368685$
1.977471
2.570857
2.496821
$-1.130567$
$-0.730609$
$-0.426162$
$-1.556837$
0.595233

C $\quad-0.928579$

$\mathrm{H} \quad-1.992503$

H $\quad-0.776525$

H $\quad-0.513041$

C -1.408628

H $\quad-2.112386$

H $\quad-1.945304$

H $\quad-1.029129$

$\begin{array}{rr}-0.195032 & -1.467812 \\ -0.336414 & -1.273340 \\ 0.676078 & -2.103561 \\ -1.052431 & -1.991533 \\ 0.104945 & 1.290172 \\ 0.907022 & 1.058547 \\ -0.845738 & 1.272110 \\ 0.262921 & 2.302271\end{array}$

$-0.195032 \quad-1.467812$

$-0.336414 \quad-1.273340$

$0.676078-2.103561$

$-1.052431 \quad-1.991533$

$0.104945 \quad 1.290172$

$-0.845738 \quad 1.272110$

$0.262921 \quad 2.302271$ 


$$
v=-182.25 \mathrm{~cm}^{-1}
$$

$\begin{array}{rrrrrrrr}\mathrm{P} & -0.660793 & 0.127272 & 0.057331 & \mathrm{C} & -0.332047 & -0.669031 & -1.534371 \\ \mathrm{~S} & 3.549347 & -0.530471 & -0.480544 & \mathrm{H} & -0.703694 & -1.694291 & -1.530503 \\ \mathrm{C} & -0.179376 & 1.730913 & 0.047201 & \mathrm{H} & -0.841920 & -0.110411 & -2.320216 \\ \mathrm{H} & -0.425406 & 2.317468 & -0.829469 & \mathrm{H} & 0.738796 & -0.660861 & -1.728872 \\ \mathrm{H} & -0.192414 & 2.262941 & 0.991017 & \mathrm{C} & 0.156035 & -0.819397 & 1.366743 \\ \mathrm{C} & 3.118752 & 1.024352 & -0.436863 & \mathrm{H} & -0.276954 & -1.818169 & 1.438525 \\ \mathrm{H} & 2.358815 & 1.408191 & 0.239495 & \mathrm{H} & 1.222878 & -0.890694 & 1.152339 \\ \mathrm{H} & 3.548599 & 1.751705 & -1.124481 & \mathrm{H} & 0.012602 & -0.302262 & 2.316445 \\ \mathrm{H} & -2.018625 & -0.296972 & 0.262835 & & & & \end{array}$

\section{2e:}

$$
\begin{aligned}
& E=-460.367526729254 \mathrm{au} \\
& Z P E=0.10877598 \mathrm{au} \\
& \mathrm{G}_{\text {corr }}=0.08051489 \mathrm{au}
\end{aligned}
$$

$\begin{array}{lrrr}\mathrm{P} & -0.694509 & 0.119959 & 0.067746 \\ \mathrm{C} & -0.200249 & 1.708928 & 0.037237 \\ \mathrm{H} & -0.437445 & 2.300748 & -0.837231 \\ \mathrm{H} & -0.125232 & 2.239731 & 0.977254 \\ \mathrm{H} & -2.048477 & -0.324709 & 0.291051 \\ \mathrm{C} & -0.389565 & -0.710714 & -1.514637 \\ \mathrm{H} & -0.782577 & -1.728209 & -1.495084\end{array}$

$\begin{array}{rrrr}\mathrm{H} & -0.891544 & -0.153729 & -2.306946 \\ \mathrm{H} & 0.680319 & -0.722129 & -1.718320 \\ \mathrm{C} & 0.126603 & -0.821045 & 1.382578 \\ \mathrm{H} & -0.274944 & -1.834055 & 1.436047 \\ \mathrm{H} & 1.198717 & -0.848645 & 1.192950 \\ \mathrm{H} & -0.048111 & -0.320931 & 2.335978\end{array}$

\section{$\operatorname{TS}(\mathbf{1 e} \rightarrow \mathbf{4}+\mathbf{5 e}):$}

5e:

$\begin{array}{lr}\mathrm{P} & 0.330383 \\ \mathrm{~S} & 2.320151 \\ \mathrm{C} & 0.719821 \\ \mathrm{H} & 0.432624 \\ \mathrm{H} & 0.116778 \\ \mathrm{C} & 2.104154 \\ \mathrm{H} & 2.520953 \\ \mathrm{H} & 2.809646 \\ \mathrm{H} & -0.623726\end{array}$
$-0.111231$
0.042034
1.992503
1.902731
2.689781
1.941419
2.581913
1.701195
0.668024

$$
\begin{aligned}
& E=-897.312975772154 \mathrm{au} \\
& Z P E=0.13977536 \mathrm{au} \\
& \mathrm{G}_{\text {corr }}=0.10786117 \mathrm{au} \\
& v=-562.91 \mathrm{~cm}^{-1}
\end{aligned}
$$

$$
\begin{array}{r}
0.330461 \\
0.750715 \\
-0.708264 \\
-1.749517 \\
-0.136473 \\
-0.411542 \\
0.354300 \\
-1.196066 \\
0.998315
\end{array}
$$

$\begin{array}{rrrr}\mathrm{C} & -0.260129 & -0.679591 & -1.304360 \\ \mathrm{H} & -0.472187 & -1.749173 & -1.285449 \\ \mathrm{H} & -1.161328 & -0.123802 & -1.562024 \\ \mathrm{H} & 0.503329 & -0.468919 & -2.048589 \\ \mathrm{C} & -0.008300 & -1.658826 & 1.314146 \\ \mathrm{H} & -1.063245 & -1.921349 & 1.202948 \\ \mathrm{H} & 0.601926 & -2.492495 & 0.969444 \\ \mathrm{H} & 0.203742 & -1.483931 & 2.368566\end{array}$

$\begin{array}{lrrr}\mathrm{P} & 0.021168 & 0.025538 & -0.114162 \\ \mathrm{H} & -0.170795 & -0.206138 & 1.267144 \\ \mathrm{C} & 1.833757 & -0.046174 & -0.254058 \\ \mathrm{H} & 2.307759 & 0.623983 & 0.465690 \\ \mathrm{H} & 2.159797 & -1.069976 & -0.075175 \\ \mathrm{H} & 2.118264 & 0.237640 & -1.267479\end{array}$

0.237640

$-1.267479$
$\mathrm{E}=-818.945343931856 \mathrm{au}$

$\mathrm{ZPE}=0.08680618 \mathrm{au}$

$\mathrm{G}_{\text {corr }}=0.05819619 \mathrm{au}$

$\mathrm{H}_{\text {corr }}=0.08957715 \mathrm{au}$ 


$$
\begin{aligned}
& E=-892.79919796177 \mathrm{au} \\
& Z P E=0.06892100 \mathrm{au} \\
& G_{\text {corr }}=0.04015340 \mathrm{au} \\
& v=-458.42 \mathrm{~cm}^{-1}
\end{aligned}
$$

$\begin{array}{lrrrrrrr}\mathrm{P} & -0.496819 & 0.156323 & 0.465940 & \mathrm{H} & -1.758553 & -2.021771 & -0.148894 \\ \mathrm{H} & -1.018597 & 1.315800 & -0.145207 & \mathrm{C} & -0.375673 & -1.153358 & -1.527557 \\ \mathrm{O} & 0.885958 & -0.140061 & -0.074751 & \mathrm{H} & 0.348620 & -1.950329 & -1.469107 \\ \mathrm{C} & -1.533001 & -1.139707 & -0.734414 & \mathrm{H} & -0.220587 & -0.425511 & -2.311688 \\ \mathrm{H} & -2.392893 & -0.574581 & -1.073528 & \mathrm{~S} & -1.024361 & 0.001923 & 2.298775\end{array}$

6:

$\operatorname{TS}\left(\mathbf{6} \rightarrow \mathbf{3}^{\circ}+\mathbf{2 d}\right):$
0.149801
1.445339
$-0.216215$
$-1.019754$
$-0.621664$

$\mathrm{E}=$
$\mathrm{ZPE}$
$\mathrm{G}_{\text {corr }}$

336
655
614
437

H $\quad-1.816704$

C $\quad-0.135176$

H 0.333191

H $\quad-0.107260$

S $\quad-0.739224$ $\begin{array}{lr}-1.952780 & -0.212957 \\ -1.051520 & -1.359097 \\ -2.032931 & -1.401789 \\ -0.579653 & -2.340517 \\ -0.051894 & 2.395300\end{array}$

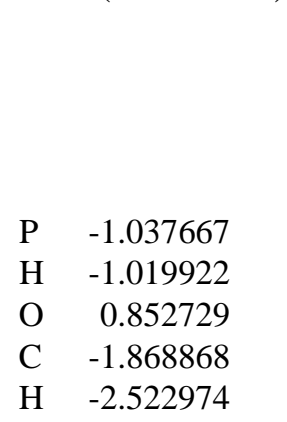

0.093711

1.332301

$-0.404396$

$-1.026867$

$-0.638524$

\begin{tabular}{|c|c|c|c|c|c|c|c|}
\hline \multicolumn{2}{|c|}{$3^{\mathbf{O}}$ (HCHO): } & & & 6 a & & & \\
\hline J & -2.969146 & -0.068187 & 1.583579 & $\mathrm{H}$ & -2.695098 & 1.343361 & 3.000098 \\
\hline c & -2.795503 & 0.276553 & 2.718864 & $\mathrm{H}$ & -2.725379 & -0.451750 & 3.550836 \\
\hline
\end{tabular}

$$
\begin{array}{r}
0.470963 \\
-0.188947 \\
-0.384432 \\
-0.470035 \\
-1.240312
\end{array}
$$

$$
\begin{aligned}
& E=-892.74235208364 \mathrm{au} \\
& Z P E=0.06534619 \mathrm{au} \\
& \mathrm{G}_{\mathrm{corr}}=0.03576639 \mathrm{au} \\
& v=-557.31 \mathrm{~cm}^{-1}
\end{aligned}
$$

$\begin{array}{lrrr}\mathrm{H} & -2.134006 & -1.970979 & -0.014330 \\ \mathrm{C} & 0.321880 & -0.959538 & -1.384674 \\ \mathrm{H} & 0.615480 & -1.976760 & -1.667211 \\ \mathrm{H} & -0.200087 & -0.391572 & -2.157135 \\ \mathrm{~S} & -0.592470 & 0.011352 & 2.315681\end{array}$

$\begin{array}{ll}\mathrm{TS}(\mathbf{1 c} \rightarrow \mathbf{4 + 7 c}): & \mathrm{E}=-892.72665426279 \\ \mathrm{ZPE}=0.06581486 \mathrm{au} \\ \mathrm{G}_{\mathrm{corr}}=0.03521008 \mathrm{au} \\ v=-414.90 \mathrm{~cm}^{-1}\end{array}$

P $\quad-0.221404$

0.152528 


$\begin{array}{lrrrrrrr}\mathrm{S} & 1.684619 & 0.527310 & -0.653240 & \mathrm{C} & 1.584816 & -2.173053 & -0.380690 \\ \mathrm{C} & 0.237529 & -2.341941 & -0.465924 & \mathrm{H} & 2.202240 & -2.162005 & -1.267818 \\ \mathrm{H} & -0.353690 & -2.569826 & 0.411973 & \mathrm{H} & 2.092994 & -2.192650 & 0.573721 \\ \mathrm{H} & -0.251064 & -2.459993 & -1.423019 & \mathrm{O} & -0.251660 & 1.667405 & -0.417274\end{array}$

\begin{tabular}{|c|c|c|c|c|c|c|}
\hline $\mathrm{TS}(5 \mathrm{c} \rightarrow 7 \mathrm{c})$ & & $\begin{array}{l}\mathrm{E} \\
\mathrm{ZH} \\
\mathrm{G}_{0} \\
\mathrm{v}=\end{array}$ & $35 \mathrm{a}$ & & & \\
\hline-0.124665 & 0.511984 & 0.198120 & $\mathrm{~S}$ & 0.868332 & 1.086171 & -1.523746 \\
\hline 0.929067 & 0.024030 & 1.059175 & $\mathrm{O}$ & -0.385233 & 2.014447 & 0.287852 \\
\hline
\end{tabular}

7c:

$E=-814.323566838088 \mathrm{au}$

$\mathrm{ZPE}=0.01335020 \mathrm{au}$

$\mathrm{G}_{\text {corr }}=-0.01180649 \mathrm{au}$

P $\quad-0.230050$

$0.300961 \quad 0.242331$

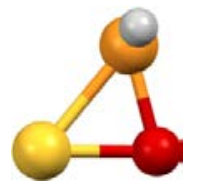

H 0.918689

$0.104615 \quad 1.077287$

S $\quad 0.731395$

1.277248

$-1.335527$

$\begin{array}{llll}\mathrm{O} & -0.132532 & 1.953809 & 0.037310\end{array}$

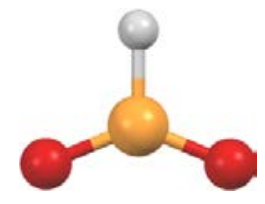

5':

$\mathrm{E}=-491.797881031961 \mathrm{au}$

$\mathrm{ZPE}=0.01632273 \mathrm{au}$

$\mathrm{G}_{\text {corr }}=-0.00763921 \mathrm{au}$

$\mathrm{H} \quad 1.366464 \quad 0.001929$

0.004162

O $\quad-0.600882$

O $\quad-0.594526$

1.341449

0.006273

$-0.015592$

$-1.34530$

$-0.053523$

\begin{tabular}{|c|c|c|c|c|c|c|}
\hline $\mathrm{TS}\left(5^{\prime} \rightarrow 7^{\prime}\right)$ & & $\begin{array}{l}\mathrm{E} \\
\mathrm{ZF} \\
\mathrm{G}_{0} \\
v=\end{array}$ & $1 \mathrm{a}$ & & & \\
\hline-0.124665 & 0.511984 & 0.198120 & $\mathrm{~S}$ & 0.868332 & 1.086171 & -1.523746 \\
\hline 0.929067 & 0.024030 & 1.059175 & $\mathrm{O}$ & -0.385233 & 2.014447 & 0.287852 \\
\hline
\end{tabular}


7':

$\operatorname{TS}(\mathbf{5 d} \rightarrow \mathbf{7 d})$ :

$\begin{array}{lll}\mathrm{P} & 1.039589 & 0.248304\end{array}$

$\mathrm{H} \quad 0.245748 \quad-0.109940$
$E=-491.656282632600 \mathrm{au}$

$\mathrm{ZPE}=0.01461166 \mathrm{au}$

$\mathrm{G}_{\text {corr }}=-0.00944115 \mathrm{au}$
$\begin{array}{lrrr}\mathrm{H} & -0.004245 & -0.008315 & -0.006998 \\ \mathrm{P} & 1.434299 & 0.013044 & 0.003117\end{array}$
O 1.672350

O 1.691486

1.648402

1.022854
0.101572

$-1.283324$

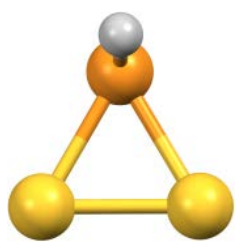

7d:

0.449649

1.582914

S $\quad 0.138584$

S $\quad 2.336764$

1.603394

1.678538

$-0.691083$

1.189308

$\operatorname{TS}(\mathbf{1 a} \rightarrow \mathbf{8 a}):$

$$
\begin{aligned}
& E=-817.587279689956 \mathrm{au} \\
& Z P E=0.06331027 \mathrm{au} \\
& \mathrm{G}_{\text {corr }}=0.03593897 \mathrm{au} \\
& \nu=-521.95 \mathrm{~cm}^{-1}
\end{aligned}
$$

$\begin{array}{lrrr}\mathrm{P} & -0.304132 & 0.125048 & -1.285925 \\ \mathrm{H} & -1.526836 & 0.786960 & -0.978355 \\ \mathrm{~S} & 0.459220 & 0.172318 & 0.781983 \\ \mathrm{C} & -1.353543 & -0.882404 & 0.988114 \\ \mathrm{H} & -1.980312 & -0.174568 & 1.509438\end{array}$

$\begin{array}{lrrr}\mathrm{H} & -1.811023 & -1.444290 & 0.196541 \\ \mathrm{C} & -0.130804 & -1.354429 & 1.633408 \\ \mathrm{H} & 0.303889 & -2.276849 & 1.265559 \\ \mathrm{H} & -0.063017 & -1.221256 & 2.709061\end{array}$

$1.748689-0.423814$

$1.798351 \quad 0.925020$ 
8a:

$\begin{array}{lrr}\text { TS(8a } & \\ & & \\ & & \\ & & \\ & & \\ \mathrm{P} & -0.451214 & 1.170421 \\ \mathrm{H} & 0.729395 & 1.083916 \\ \mathrm{~S} & 0.100865 & -0.708401 \\ \mathrm{C} & -1.390993 & -1.596017 \\ \mathrm{H} & -1.348161 & -2.676196\end{array}$

$\mathrm{E}=-817.629429888845 \mathrm{au}$

$\mathrm{E}=-817.608067631421 \mathrm{au}$ (NCEPA1/def2-TZVPP)

$\mathrm{ZPE}=0.06384065 \mathrm{au}$

$\mathrm{G}_{\text {corr }}=0.03605509 \mathrm{au}$

$\begin{array}{lrrr}\mathrm{P} & -0.579111 & 0.868317 & -0.930131 \\ \mathrm{H} & 0.761816 & 1.345706 & -0.960959 \\ \mathrm{~S} & 0.103254 & -0.836104 & 0.102847 \\ \mathrm{C} & -1.402690 & -1.569785 & 0.799049 \\ \mathrm{H} & -1.422176 & -2.651207 & 0.762648\end{array}$

$\begin{array}{lrrr}\mathrm{H} & -2.307312 & -1.085660 & 0.453082 \\ \mathrm{C} & -0.603691 & -0.903236 & 1.853581 \\ \mathrm{H} & -0.043437 & -1.514086 & 2.550496 \\ \mathrm{H} & -0.913212 & 0.076583 & 2.189212\end{array}$

\section{$\operatorname{TS}(\mathbf{8} \mathbf{a} \rightarrow \mathbf{5 a}+\mathbf{4 a}):$}

\begin{tabular}{lllll}
\multicolumn{6}{c}{$\mathrm{E}=-817.628159106924 \mathrm{au}$} & & \\
$\mathrm{ZPE}=0.06358367 \mathrm{au}$ & & & & \\
$\mathrm{G}_{\mathrm{corr}}=0.03637048 \mathrm{au}$ & & & & \\
$v=-166.92 \mathrm{~cm}^{-1}$ & & & & \\
& & & & \\
-0.583168 & $\mathrm{H}$ & -2.308265 & -1.139788 & 0.417376 \\
-1.370614 & $\mathrm{C}$ & -0.635453 & -0.908991 & 1.861952 \\
0.206603 & $\mathrm{H}$ & -0.064629 & -1.509036 & 2.559936 \\
0.767726 & $\mathrm{H}$ & -1.038106 & 0.014622 & 2.258379 \\
0.701636 & & & &
\end{tabular}

$$
\begin{aligned}
& E=-817.623237738026 \mathrm{au} \\
& \mathrm{ZPE}=0.06255678 \mathrm{au} \\
& \mathrm{G}_{\mathrm{corr}}=0.03453545 \mathrm{au} \\
& v=-269.99 \mathrm{~cm}^{-1}
\end{aligned}
$$

$\begin{array}{lrrr}\mathrm{P} & -0.426301 & 0.729597 & -1.176336 \\ \mathrm{H} & 0.619618 & 1.529764 & -0.632764 \\ \mathrm{~S} & 0.135421 & -0.935179 & -0.114254 \\ \mathrm{C} & -1.454878 & -1.568315 & 0.896772 \\ \mathrm{H} & -1.479229 & -2.648983 & 0.850196\end{array}$

$\begin{array}{lrrr}\mathrm{H} & -2.315883 & -1.064823 & 0.480987 \\ \mathrm{C} & -0.663162 & -0.927106 & 1.880144 \\ \mathrm{H} & -0.002093 & -1.497620 & 2.516146 \\ \mathrm{H} & -0.820052 & 0.113195 & 2.118934\end{array}$

$\operatorname{TS}(\mathbf{1 a} \rightarrow \mathbf{9 a}):$

$$
\begin{aligned}
& E=-817.616574796311 \mathrm{au} \\
& Z P E=0.06219232 \mathrm{au} \\
& G_{\text {corr }}=0.03473063 \mathrm{au} \\
& v=-177.54 \mathrm{~cm}^{-1}
\end{aligned}
$$

$\begin{array}{lrrr}\mathrm{P} & -1.810765 & 0.641284 & -0.012944 \\ \mathrm{H} & -0.458587 & 1.022076 & -0.230100 \\ \mathrm{~S} & 1.341607 & -1.127111 & 0.460016\end{array}$

$\begin{array}{lllr}\mathrm{C} & -1.429041 & -1.058113 & 0.490020 \\ \mathrm{H} & -2.291327 & -1.442583 & 1.045373 \\ \mathrm{H} & -1.228325 & -1.744292 & -0.337123\end{array}$




\begin{tabular}{|c|c|c|c|}
\hline-0.157428 & -0.928763 & 1.351413 & $\mathrm{H}$ \\
\hline-0.211536 & -1.673739 & 2.156334 & \\
\hline
\end{tabular}

9a:

$$
\mathrm{E}=-817.676450520724 \mathrm{au}
$$$$
\mathrm{ZPE}=0.06240292 \mathrm{au}
$$$$
\mathrm{G}_{\text {corr }}=0.03394073 \mathrm{au}
$$

$\begin{array}{lrrr}\mathrm{P} & -1.626345 & 0.650694 & 0.215150 \\ \mathrm{H} & -0.351046 & 0.998342 & -0.296456 \\ \mathrm{~S} & 1.408023 & -1.869038 & 0.441541 \\ \mathrm{C} & -1.266311 & -1.181624 & 0.472577 \\ \mathrm{H} & -2.093181 & -1.561745 & 1.078864\end{array}$

$\begin{array}{rrrr}\mathrm{H} & -1.289689 & -1.656533 & -0.507656 \\ \mathrm{C} & 0.033759 & -1.385446 & 1.147025 \\ \mathrm{H} & 0.030194 & -1.143910 & 2.212023 \\ \mathrm{H} & -1.309599 & 1.080020 & 1.531257\end{array}$

$\operatorname{TS}(\mathbf{1 a} \rightarrow \mathbf{1 0 a}):$

$$
\begin{aligned}
& E=-817.623898800831 \mathrm{au} \\
& Z P E=0.06397465 \mathrm{au} \\
& \mathrm{G}_{\text {corr }}=0.03665909 \mathrm{au} \\
& v=-449.21 \mathrm{~cm}^{-1}
\end{aligned}
$$

$\begin{array}{lrrr}\mathrm{P} & -0.499785 & 0.154851 & -0.412285 \\ \mathrm{H} & -0.795582 & 1.184452 & 0.537431 \\ \mathrm{~S} & 1.488488 & -0.318758 & -0.045210 \\ \mathrm{C} & -1.624987 & -1.134734 & 0.628059 \\ \mathrm{H} & -2.479727 & -0.541192 & 0.928155\end{array}$

$\begin{array}{lrrr}\mathrm{H} & -1.839418 & -2.008481 & 0.025365 \\ \mathrm{C} & -0.484551 & -1.160065 & 1.454976 \\ \mathrm{H} & 0.194319 & -1.993340 & 1.441128 \\ \mathrm{H} & -0.365315 & -0.452202 & 2.262205\end{array}$

\section{0a:}

$$
\begin{aligned}
& E=-817.686781328974 \mathrm{au} \\
& Z P E=0.06555930 \mathrm{au} \\
& G_{\text {corr }}=0.03843769 \mathrm{au}
\end{aligned}
$$

$\begin{array}{lrrr}\mathrm{P} & -0.167325 & -0.036157 & 0.181520 \\ \mathrm{H} & -0.607465 & 1.263689 & 0.479558 \\ \mathrm{~S} & 1.229518 & -0.288914 & -1.128925 \\ \mathrm{C} & -1.565100 & -1.110544 & 0.536264 \\ \mathrm{H} & -2.538527 & -0.671230 & 0.715863\end{array}$

$\begin{array}{lrrr}\mathrm{H} & -1.592725 & -2.047696 & -0.005611 \\ \mathrm{C} & -0.502973 & -1.005500 & 1.659085 \\ \mathrm{H} & 0.119376 & -1.878743 & 1.809572 \\ \mathrm{H} & -0.781339 & -0.494376 & 2.572499\end{array}$

11:

$$
\begin{aligned}
& E=-476.177069455435 \mathrm{au} \\
& E=-476.158812965860 \mathrm{au}(\mathrm{NCEPA} 1 / \text { def2-TZVPP) } \\
& Z P E=0.05483882 \mathrm{au} \\
& \mathrm{G}_{\text {corr }}=0.03059469 \mathrm{au} \\
& \mathrm{H}_{\text {corr }}=0.05916178 \mathrm{au}
\end{aligned}
$$

$\begin{array}{lrrr}\mathrm{S} & -1.230881 & 1.620068 & -0.735197 \\ \mathrm{C} & -2.080740 & 0.374863 & -1.759548 \\ \mathrm{H} & -2.892961 & 0.753733 & -2.366897 \\ \mathrm{H} & -2.281053 & -0.568350 & -1.267210\end{array}$

$\begin{array}{rrrr}\mathrm{C} & -0.694222 & 0.659817 & -2.188669 \\ \mathrm{H} & -0.545579 & 1.236099 & -3.093019 \\ \mathrm{H} & 0.066566 & -0.084997 & -1.992355\end{array}$




\section{8at:}

$$
\mathrm{E}=-817.598030168619 \mathrm{au} \text { (NCEPA1/def2-TZVPP) }
$$$$
\mathrm{ZPE}=0.06101838 \mathrm{au}
$$$$
\mathrm{G}_{\text {corr }}=0.03020254 \mathrm{au}
$$

$\begin{array}{lrrr}\mathrm{P} & -0.488356 & 0.305501 & -1.681924 \\ \mathrm{H} & 0.627251 & 1.128798 & -2.002208 \\ \mathrm{~S} & 0.203950 & 0.047139 & 0.272329 \\ \mathrm{C} & -1.142578 & -1.083982 & 0.945642 \\ \mathrm{H} & -1.098625 & -1.993486 & 0.348464\end{array}$

$\begin{array}{llll}\mathrm{H} & -2.084447 & -0.568332 & 0.761593 \\ \mathrm{C} & -0.909023 & -1.334705 & 2.370983 \\ \mathrm{H} & -0.263815 & -2.141168 & 2.689794 \\ \mathrm{H} & -1.250917 & -0.629236 & 3.115152\end{array}$

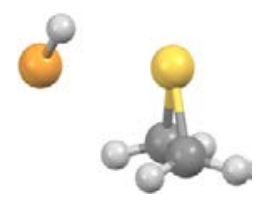

\section{8a $\mathbf{a}^{\mathrm{MECP}}$ :}

P $\quad-0.654917$

H 0.703849

S 0.232050

C $\quad-1.414386$

H -1.578826
1.138889

1.554147

$-1.210283$

$-1.643765$

$-2.704776$
$-1.009032$

$-0.892348$

0.174941

0.824513

0.956937
$E=-817.573983844234 \mathrm{au}$ (NCEPA1/def2-TZVPP)

$\mathrm{ZPE}=0.06173350 \mathrm{au}$

$\mathrm{G}_{\text {corr }}=0.03094226 \mathrm{au}$

\section{$\operatorname{TS}\left(\mathbf{8} a^{t} \rightarrow \mathbf{1 1}+12 a^{t}\right):$}

$\begin{array}{lll}\text { P } & -0.654917 & 1.138889\end{array}$

$\begin{array}{lll}\mathrm{H} & 0.703849 & 1.554147\end{array}$

S $0.232050 \quad-1.210283$

C $-1.414386-1.643765$

H $-1.578826 \quad-2.704776$

$\begin{array}{llll}\mathrm{H} & -2.224478 & -1.095767 & 0.359431 \\ \mathrm{C} & -0.562293 & -0.931028 & 1.796155 \\ \mathrm{H} & -0.125840 & -1.487623 & 2.615312 \\ \mathrm{H} & -0.781718 & 0.110737 & 1.993916\end{array}$

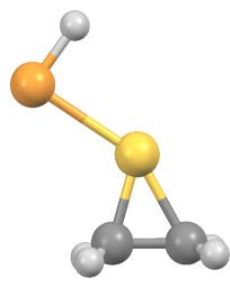

$\mathrm{E}=-817.564599132715 \mathrm{au}$ (NCEPA1/def2-TZVPP)

$\mathrm{ZPE}=0.06076857 \mathrm{au}$

$\mathrm{G}_{\text {corr }}=0.03039986 \mathrm{au}$

$v=-341.23 \mathrm{~cm}^{-1}$

$\begin{array}{lrrr}\mathrm{H} & -2.224478 & -1.095767 & 0.359431 \\ \mathrm{C} & -0.562293 & -0.931028 & 1.796155 \\ \mathrm{H} & -0.125840 & -1.487623 & 2.615312 \\ \mathrm{H} & -0.781718 & 0.110737 & 1.993916\end{array}$




\section{1·12at:}

\section{2as:}

12at:

$\operatorname{TS}(\mathbf{1 b} \rightarrow \mathbf{8 b}):$

$$
\mathrm{E}=-817.583635340011 \mathrm{au} \text { (NCEPA1/def2-TZVPP) }
$$$$
\mathrm{ZPE}=0.06163776 \mathrm{au}
$$$$
\mathrm{G}_{\text {corr }}=0.02897730 \mathrm{au}
$$

$\begin{array}{lrrr}\mathrm{P} & -0.520577 & 1.437191 & -0.954005 \\ \mathrm{H} & 0.740458 & 1.287839 & -1.600950 \\ \mathrm{~S} & 0.319525 & -1.434293 & 0.402805 \\ \mathrm{C} & -1.426913 & -1.630283 & 0.889155 \\ \mathrm{H} & -1.729847 & -2.648935 & 1.093650\end{array}$

P

0.256820

$-0.337953$

$-0.091519$

$$
\begin{aligned}
& E=-341.380057317151 \mathrm{au} \\
& E=-341.804566506064 \mathrm{au}(\text { NCEPA1/def2-TZVPP) } \\
& Z P E=0.00536319 \mathrm{au} \\
& \mathrm{G}_{\text {corr }}=-0.01189675 \mathrm{au}
\end{aligned}
$$

$\begin{array}{llll}\mathrm{H} & -2.124842 & -1.046701 & 0.302552\end{array}$

$\begin{array}{llll}\text { C } & -0.602659 & -0.925896 & 1.891968\end{array}$

$\begin{array}{llll}\mathrm{H} & -0.328994 & -1.452046 & 2.797269\end{array}$

$\begin{array}{llll}\mathrm{H} & -0.732711 & 0.143653 & 1.997380\end{array}$

$$
\begin{aligned}
& \mathrm{E}=-341.424276639186 \mathrm{au} \text { (NCEPA1/def2-TZVPP) } \\
& \mathrm{ZPE}=0.00532479 \mathrm{au} \\
& \mathrm{G}_{\text {corr }}=-0.01297469 \mathrm{au} \\
& \begin{array}{llll}
\mathrm{P} & 0.257074 & -0.337210 & -0.091833
\end{array} \\
& \begin{array}{llll}
\text { H } & -0.172082 & -1.592835 & 0.438670
\end{array}
\end{aligned}
$$$$
\begin{aligned}
& E=-1451.3714795866 \\
& Z P E=0.10447649 \mathrm{au} \\
& G_{\text {corr }}=0.05861364 \mathrm{au} \\
& v=-281.71 \mathrm{~cm}^{-1}
\end{aligned}
$$

$\begin{array}{lrrr}\text { Mo } & 0.062497 & -0.132886 & 2.846078 \\ \text { C } & 0.375470 & 0.478402 & 4.756409 \\ \text { O } & 0.570146 & 0.826817 & 5.828165 \\ \text { C } & 1.443175 & 1.249711 & 2.183641 \\ \text { O } & 2.201707 & 2.008803 & 1.798167 \\ \text { C } & -1.440171 & 1.259958 & 2.586570 \\ \text { O } & -2.274843 & 2.023761 & 2.440770 \\ \text { C } & -1.325623 & -1.518820 & 3.499216 \\ \text { O } & -2.087112 & -2.278658 & 3.878751 \\ \text { C } & 1.540115 & -1.551836 & 3.052725\end{array}$

$\begin{array}{lrrr}\mathrm{O} & 2.353909 & -2.345308 & 3.155667 \\ \mathrm{P} & -0.181179 & -0.859478 & 0.489089 \\ \mathrm{H} & 0.537942 & -1.947263 & -0.076769 \\ \mathrm{~S} & 0.053008 & 0.493657 & -1.050339 \\ \mathrm{C} & -0.484993 & -0.737224 & -2.334463 \\ \mathrm{H} & -0.277475 & -0.357128 & -3.340890 \\ \mathrm{H} & 0.148592 & -1.618352 & -2.182071 \\ \mathrm{C} & -1.909976 & -0.864236 & -2.072574 \\ \mathrm{H} & -2.596741 & -1.076672 & -2.884263 \\ \mathrm{H} & -2.326463 & -0.607760 & -1.115697\end{array}$

8b:

$$
\begin{aligned}
& E=-1451.40624937272 \mathrm{au} \\
& Z P E=0.10708977 \mathrm{au}
\end{aligned}
$$


$\mathrm{G}_{\text {corr }}=0.05945338 \mathrm{au}$

$\begin{array}{lrrr}\text { Mo } & 0.097652 & -0.289671 & 2.700849 \\ \text { C } & 0.024806 & 0.722430 & 4.442172 \\ \text { O } & -0.008201 & 1.299475 & 5.433087 \\ \text { C } & 1.424432 & 1.070939 & 1.921430 \\ \text { O } & 2.166331 & 1.824745 & 1.483444 \\ \text { C } & -1.474040 & 0.774547 & 1.943583 \\ \text { O } & -2.353156 & 1.354011 & 1.485565 \\ \text { C } & -1.229757 & -1.676049 & 3.448015 \\ \text { O } & -1.974490 & -2.436527 & 3.864762 \\ \text { C } & 1.689728 & -1.414247 & 3.373718\end{array}$

$\begin{array}{lrrr}\mathrm{O} & 2.574718 & -2.042992 & 3.729819 \\ \mathrm{P} & 0.184795 & -1.738278 & 0.555673 \\ \mathrm{H} & 1.571063 & -1.844118 & 0.248340 \\ \mathrm{~S} & 0.078888 & -0.154601 & -0.987849 \\ \mathrm{C} & -0.856494 & -0.844261 & -2.428364 \\ \mathrm{H} & -0.598034 & -0.383314 & -3.372711 \\ \mathrm{H} & -0.894725 & -1.924991 & -2.411040 \\ \mathrm{C} & -1.690927 & -0.099124 & -1.468912 \\ \mathrm{H} & -2.022727 & 0.901075 & -1.710489 \\ \mathrm{H} & -2.327874 & -0.653560 & -0.792909\end{array}$

$\mathrm{TS}\left(\mathbf{8 b} \rightarrow \mathbf{5} \mathbf{b}^{\prime}+\mathbf{4}\right)$ :

$$
\begin{aligned}
& E=-1451.38968705168 \mathrm{au} \\
& Z P E=0.10534645 \mathrm{au} \\
& \mathrm{G}_{\text {corr }}=0.05781310 \mathrm{au} \\
& v=-308.86 \mathrm{~cm}^{-1}
\end{aligned}
$$

$\begin{array}{lrrr}\text { Mo } & 0.232934 & -0.115614 & 2.658539 \\ \text { C } & 0.192989 & 0.667705 & 4.505217 \\ \text { O } & 0.184302 & 1.106372 & 5.564288 \\ \text { C } & 1.941204 & 0.913447 & 2.153644 \\ \text { O } & 2.883913 & 1.474740 & 1.836454 \\ \text { C } & -0.887900 & 1.524550 & 2.116510 \\ \text { O } & -1.503545 & 2.468526 & 1.919568 \\ \text { C } & -1.481932 & -1.200658 & 2.978327 \\ \text { O } & -2.435143 & -1.811371 & 3.136078 \\ \text { C } & 1.333648 & -1.663812 & 3.469155\end{array}$

$\begin{array}{lrrr}\mathrm{O} & 1.922809 & -2.505938 & 3.964207 \\ \mathrm{P} & 0.617524 & -1.506220 & 0.425291 \\ \mathrm{H} & 1.667734 & -0.762474 & -0.183773 \\ \mathrm{~S} & -0.905000 & -0.256404 & -0.284869 \\ \mathrm{C} & -1.092183 & -0.552283 & -2.425117 \\ \mathrm{H} & -1.403340 & 0.399023 & -2.832573 \\ \mathrm{H} & -0.131166 & -0.932615 & -2.738009 \\ \mathrm{C} & -1.985133 & -1.354912 & -1.712155 \\ \mathrm{H} & -3.018296 & -1.058657 & -1.594336 \\ \mathrm{H} & -1.751428 & -2.387917 & -1.498262\end{array}$

5b':

$$
\begin{aligned}
& E=-1372.98168381294 \mathrm{au} \\
& Z P E=0.05386250 \mathrm{au} \\
& G_{\text {corr }}=0.00842156 \mathrm{au}
\end{aligned}
$$

$\begin{array}{lrrr}\text { Mo } & 0.211475 & 0.011637 & 3.007838 \\ \text { C } & 0.427933 & 0.689596 & 4.885582 \\ \text { O } & 0.556530 & 1.081438 & 5.952137 \\ \text { C } & 1.927331 & 0.995479 & 2.394601 \\ \text { O } & 2.873592 & 1.526609 & 2.050043 \\ \text { C } & -0.928391 & 1.731762 & 2.640226 \\ \text { O } & -1.543642 & 2.673751 & 2.485294\end{array}$

C $\quad-1.515781$

$-1.045649$

3.467287

O $\quad-2.455791$

$-1.637304$

3.713175

C 1.328416

$-1.598944$

3.677209

O 1.933722

$-2.455274$

4.123734

P $\quad 0.675357$

$-1.794143$

0.709215

$\mathrm{H} \quad 1.955177 \quad-1.230562 \quad 0.392154$

O -1.543642

S $\quad-0.540851$

$-0.258763$

0.239274

\author{
$\mathrm{TS}(\mathbf{1 b} \rightarrow \mathbf{9 b}):$
}

$$
\begin{aligned}
& E=-1451.36491328661 \mathrm{au} \\
& Z P E=0.10448395 \mathrm{au} \\
& \mathrm{G}_{\text {corr }}=0.05839013 \mathrm{au} \\
& v=-170.05 \mathrm{~cm}^{-1}
\end{aligned}
$$

$\begin{array}{llll}\text { Mo } & 0.143127 & -0.406033 & 2.588036 \\ \text { C } & 0.377966 & -0.195160 & 4.619015 \\ \text { O } & 0.521633 & -0.051916 & 5.740097\end{array}$

$\begin{array}{lrrr}\mathrm{C} & 1.737318 & 0.895984 & 2.371731 \\ \mathrm{O} & 2.624346 & 1.600400 & 2.264515 \\ \mathrm{C} & -1.232752 & 1.125498 & 2.571109\end{array}$




$\begin{array}{rrrrrrrr}\mathrm{O} & -2.012622 & 1.955782 & 2.540003 & \mathrm{~S} & -0.254111 & -0.515149 & -3.404999 \\ \mathrm{C} & -1.450001 & -1.709751 & 2.890771 & \mathrm{C} & -1.188074 & -0.861165 & -1.976745 \\ \mathrm{O} & -2.334985 & -2.400922 & 3.072015 & \mathrm{H} & -2.178915 & -1.285710 & -2.161955 \\ \mathrm{C} & 1.500608 & -1.966234 & 2.516621 & \mathrm{H} & -0.676589 & -1.743181 & -1.436644 \\ \mathrm{O} & 2.257766 & -2.814496 & 2.463807 & \mathrm{C} & -1.261154 & 0.259272 & -0.924204 \\ \mathrm{P} & -0.014577 & -0.427595 & 0.189722 & \mathrm{H} & -2.219810 & 0.307049 & -0.404857 \\ \mathrm{H} & 1.065217 & -0.554665 & -0.710099 & \mathrm{H} & -1.022400 & 1.233481 & -1.349755\end{array}$

9b:

$E=-1451.44236423801 \mathrm{au}$

$\mathrm{ZPE}=0.10554740 \mathrm{au}$

$\mathrm{G}_{\text {corr }}=0.05845183 \mathrm{au}$

$\begin{array}{lrrr}\text { Mo } & 0.284340 & -0.284566 & 2.490693 \\ \text { C } & 0.415971 & -0.094024 & 4.503939 \\ \text { O } & 0.486909 & 0.013021 & 5.640001 \\ \text { C } & 1.943157 & 0.916244 & 2.243744 \\ \text { O } & 2.865633 & 1.571462 & 2.096278 \\ \text { C } & -0.947417 & 1.360321 & 2.401905 \\ \text { O } & -1.641359 & 2.266207 & 2.349337 \\ \text { C } & -1.366336 & -1.490028 & 2.690454 \\ \text { O } & -2.291011 & -2.155833 & 2.784279 \\ \text { C } & 1.518930 & -1.931667 & 2.545499\end{array}$

$\begin{array}{lrrr}\mathrm{O} & 2.209876 & -2.840121 & 2.563985 \\ \mathrm{P} & 0.096396 & -0.514973 & 0.007647 \\ \mathrm{H} & 0.732094 & 0.416017 & -0.839385 \\ \mathrm{~S} & -1.600283 & 0.468341 & -3.321045 \\ \mathrm{C} & -1.583544 & -0.684284 & -2.190707 \\ \mathrm{H} & -1.501670 & -1.732304 & -2.484918 \\ \mathrm{H} & 0.588999 & -1.683360 & -0.613962 \\ \mathrm{C} & -1.615952 & -0.455123 & -0.724798 \\ \mathrm{H} & -2.192450 & -1.225962 & -0.207943 \\ \mathrm{H} & -2.020295 & 0.526120 & -0.476820\end{array}$

$\mathrm{TS}(\mathbf{8 b} \rightarrow \mathbf{1 1}+\mathbf{1 2 b}):$

$$
\begin{aligned}
& E=-1451.3767443606 \mathrm{au} \\
& Z P E=0.10386768 \mathrm{au} \\
& \mathrm{G}_{\text {corr }}=0.05721100 \mathrm{au} \\
& v=-55.72 \mathrm{~cm}^{-1}
\end{aligned}
$$

$\begin{array}{lrrr}\text { Mo } & 0.046768 & -0.522569 & 2.748741 \\ \text { C } & 0.267741 & 0.973920 & 4.209591 \\ \text { O } & 0.406700 & 1.791920 & 4.988159 \\ \text { C } & 1.286864 & 0.601079 & 1.509580 \\ \text { O } & 2.007203 & 1.193374 & 0.860142 \\ \text { C } & -1.696330 & 0.357760 & 2.064715 \\ \text { O } & -2.685675 & 0.798213 & 1.713167 \\ \text { C } & -1.159810 & -1.627540 & 4.036862 \\ \text { O } & -1.835205 & -2.223886 & 4.731579 \\ \text { C } & 1.740542 & -1.551940 & 3.317002\end{array}$

$\begin{array}{lrrr}\mathrm{O} & 2.674631 & -2.146679 & 3.586650 \\ \mathrm{P} & -0.234934 & -2.096731 & 0.981976 \\ \mathrm{H} & 1.101478 & -2.505853 & 0.671205 \\ \mathrm{~S} & -0.804392 & 1.306459 & -1.284239 \\ \mathrm{C} & -0.375366 & -0.203877 & -2.206540 \\ \mathrm{H} & -0.082047 & -0.048193 & -3.236801 \\ \mathrm{H} & 0.229681 & -0.922974 & -1.666435 \\ \mathrm{C} & -1.807594 & -0.103304 & -1.855046 \\ \mathrm{H} & -2.515799 & 0.125959 & -2.640715 \\ \mathrm{H} & -2.182466 & -0.749652 & -1.071409\end{array}$

12b:

$$
\begin{aligned}
& E=-975.19461227825 \mathrm{au} \\
& Z P E=0.04848606 \mathrm{au} \\
& G_{\text {corr }}=0.00588394 \mathrm{au}
\end{aligned}
$$

$\begin{array}{lrrr}\text { P } & -0.081772 & -2.133584 & 1.047695 \\ \text { H } & 1.209153 & -2.749734 & 1.014501 \\ \text { Mo } & 0.087942 & -0.519766 & 2.802982 \\ \text { C } & 0.180969 & 0.932104 & 4.317934 \\ \text { O } & 0.241740 & 1.718644 & 5.138079 \\ \text { C } & 1.322799 & 0.696880 & 1.646196 \\ \text { O } & 1.998838 & 1.362205 & 1.019408\end{array}$

$\begin{array}{rrrr}\mathrm{C} & -1.594385 & 0.328220 & 1.968547 \\ \mathrm{O} & -2.520570 & 0.780327 & 1.481930 \\ \mathrm{C} & -1.139707 & -1.661667 & 4.050603 \\ \mathrm{O} & -1.814233 & -2.278622 & 4.726026 \\ \mathrm{C} & 1.766792 & -1.531537 & 3.426446 \\ \mathrm{O} & 2.696253 & -2.124438 & 3.715579\end{array}$




$$
E=-892.74961077086 \mathrm{au}
$$

$$
\begin{aligned}
& \mathrm{ZPE}=0.06682517 \mathrm{au} \\
& \mathrm{G}_{\text {corr }}=0.03721594 \mathrm{au} \\
& v=-146.06 \mathrm{~cm}^{-1}
\end{aligned}
$$

$\begin{array}{lrrrrrrr}\mathrm{P} & -0.530772 & -0.128743 & 0.136086 & \mathrm{H} & 0.506419 & -0.929449 & -1.884346 \\ \mathrm{H} & 0.460360 & 0.709216 & 0.692337 & \mathrm{C} & 1.645522 & -1.734843 & -0.160113 \\ \mathrm{~S} & 3.080146 & -1.124826 & -0.947790 & \mathrm{H} & 1.598799 & -2.828109 & -0.224162 \\ \mathrm{C} & 0.274362 & -1.338197 & -0.906117 & \mathrm{H} & 1.585036 & -1.494340 & 0.908189 \\ \mathrm{H} & -0.405373 & -2.185989 & -1.012959 & \mathrm{O} & -1.617135 & -0.496348 & 1.070732\end{array}$

13c:

$$
\mathrm{TS}(\mathbf{1 3 c} \rightarrow \mathbf{2 c}+\mathbf{3}):
$$
$-0.332429$
0.869383
$-2.130749$
$-1.126715$
$-1.928189$
0.121079
0.538049
$-1.249128$
$-1.015883$
$-1.587457$

$$
\begin{aligned}
& E=-892.76397877454 \mathrm{au} \\
& Z P E=0.06820219 \mathrm{au} \\
& G_{\text {corr }}=0.03811739 \mathrm{au}
\end{aligned}
$$

H $\quad 0.837271$

C $\quad 1.575972$

H 1.120648

H 1.913612

O -1.233510
$-0.334373$

$-1.699256$

$-2.525344$

$-0.936438$

$-1.129097$
$-1.664911$

$-0.030823$

0.514317

0.668694

1.277588

$$
\begin{aligned}
& E=-892.748291236811 \mathrm{au} \\
& Z P E=0.06611770 \mathrm{au} \\
& \mathrm{G}_{\text {corr }}=0.03568550 \mathrm{au} \\
& v=-470.25 \mathrm{~cm}^{-1}
\end{aligned}
$$

$\begin{array}{lrrr}\mathrm{P} & -0.749232 & -0.329434 & 0.145657 \\ \mathrm{H} & -0.168360 & 0.879249 & 0.593858 \\ \mathrm{~S} & 2.858438 & -2.275453 & -1.243117 \\ \mathrm{C} & 0.304812 & -1.023860 & -1.011720 \\ \mathrm{H} & -0.047537 & -1.913539 & -1.516922\end{array}$

$\begin{array}{lrrr}\mathrm{H} & 0.757736 & -0.283586 & -1.662975 \\ \mathrm{C} & 1.820811 & -1.785071 & -0.017562 \\ \mathrm{H} & 1.233965 & -2.525481 & 0.522744 \\ \mathrm{H} & 2.110149 & -0.939090 & 0.601445 \\ \mathrm{O} & -1.519440 & -1.076940 & 1.160117\end{array}$

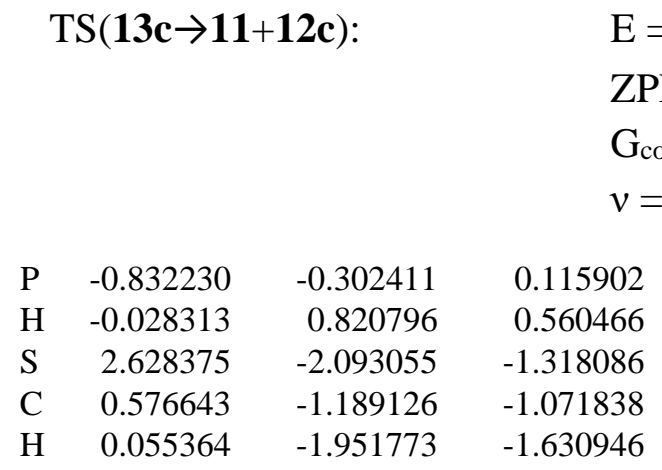

$E=-892.757190339468 \mathrm{au}$

$$
\begin{aligned}
& \mathrm{ZPE}=0.06714333 \mathrm{au} \\
& \mathrm{G}_{\text {corr }}=0.03710631 \mathrm{au} \\
& v=-356.77 \mathrm{~cm}^{-1}
\end{aligned}
$$

$\begin{array}{lrrr}\mathrm{H} & 0.876296 & -0.316255 & -1.643153 \\ \mathrm{C} & 1.538242 & -1.691438 & 0.012611 \\ \mathrm{H} & 1.101466 & -2.521047 & 0.561469 \\ \mathrm{H} & 1.897215 & -0.915421 & 0.681550 \\ \mathrm{O} & -1.211716 & -1.113476 & 1.303550\end{array}$

$\operatorname{TS}(\mathbf{1 c} \rightarrow \mathbf{1 4}):$

$E=-892.756415783438 \mathrm{au}$

$\mathrm{ZPE}=0.06751770 \mathrm{au}$ 
$\mathrm{G}_{\text {corr }}=0.03863709 \mathrm{au}$

$v=-494.87 \mathrm{~cm}^{-1}$

$\begin{array}{lrrrrrrr}\mathrm{P} & -0.532207 & -0.153852 & -0.055038 & \mathrm{H} & -0.351763 & -2.335717 & -1.109246 \\ \mathrm{H} & -0.576797 & 0.162895 & 1.326757 & \mathrm{C} & 1.657275 & -1.632125 & -0.504558 \\ \mathrm{~S} & 2.275101 & -0.060425 & 0.121853 & \mathrm{H} & 1.954292 & -1.773366 & -1.544030 \\ \mathrm{C} & 0.139851 & -1.828652 & -0.277036 & \mathrm{H} & 2.141354 & -2.418910 & 0.081823 \\ \mathrm{H} & -0.073072 & -2.376749 & 0.642856 & \mathrm{O} & -0.036669 & 0.865275 & -1.011523\end{array}$

14:

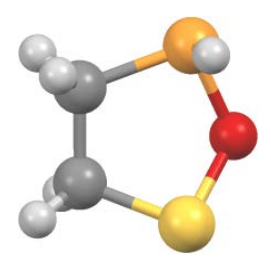

$E=-892.825653881981 \mathrm{au}$

$\mathrm{ZPE}=0.07050631 \mathrm{au}$

$\mathrm{G}_{\text {corr }}=0.04209493 \mathrm{au}$

$\begin{array}{lrrr}\mathrm{P} & -0.739207 & -0.135052 & -0.213198 \\ \mathrm{H} & -0.650354 & 0.004205 & 1.204509 \\ \mathrm{~S} & 2.053002 & 0.076463 & -0.051153 \\ \mathrm{C} & 0.097804 & -1.821538 & -0.242212 \\ \mathrm{H} & -0.062434 & -2.312220 & 0.717257\end{array}$

$\begin{array}{llll}\mathrm{H} & -0.344853 & -2.440800 & -1.022346\end{array}$

$\begin{array}{llll}\text { C } & 1.597646 & -1.614739 & -0.530095\end{array}$

$\mathrm{H} \quad 1.806925 \quad-1.750277 \quad-1.590799$

$\mathrm{H} \quad 2.226609 \quad-2.295648 \quad 0.044546$

$\begin{array}{llll}\text { O } & 0.612226 & 0.737978 & -0.644652\end{array}$

$\mathrm{TS}(\mathbf{1 4} \rightarrow \mathbf{1 5})$ :

$$
\begin{aligned}
& E=-892.741335578462 \mathrm{au} \\
& \mathrm{ZPE}=0.06832282 \mathrm{au} \\
& \mathrm{G}_{\text {corr }}=0.04054767 \mathrm{au} \\
& v=-520.63 \mathrm{~cm}^{-1}
\end{aligned}
$$

$\begin{array}{lrrr}\mathrm{P} & 0.178233 & 0.096923 & -0.179201 \\ \mathrm{H} & 0.063258 & -0.013125 & 1.285205 \\ \mathrm{~S} & 2.448637 & -0.033276 & -0.074990 \\ \mathrm{C} & 0.467864 & -1.750393 & -0.267404 \\ \mathrm{H} & -0.211138 & -2.322612 & 0.361245\end{array}$

$\begin{array}{lrrr}\mathrm{H} & 0.386897 & -2.080228 & -1.299369 \\ \mathrm{C} & 1.921154 & -1.785160 & 0.227383 \\ \mathrm{H} & 2.581453 & -2.491878 & -0.273310 \\ \mathrm{H} & 1.973304 & -1.926722 & 1.307710 \\ \mathrm{O} & 1.672684 & 0.223172 & -1.496810\end{array}$

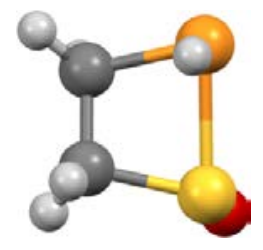

15:

$$
\begin{aligned}
& E=-892.789336612643 \mathrm{au} \\
& Z P E=0.06938296 \mathrm{au} \\
& G_{\text {corr }}=0.04069569 \mathrm{au}
\end{aligned}
$$

$\begin{array}{lrrr}\mathrm{P} & -0.072943 & 0.006605 & 0.023582 \\ \mathrm{H} & 0.059597 & 0.011573 & 1.442387 \\ \mathrm{~S} & 2.198374 & 0.062643 & -0.215036 \\ \mathrm{C} & 0.373120 & -1.809696 & -0.192159\end{array}$

$\begin{array}{lrrr}\mathrm{H} & -0.223773 & -2.504115 & 0.398426 \\ \mathrm{H} & 0.243938 & -2.054995 & -1.245815 \\ \mathrm{C} & 1.848495 & -1.727915 & 0.180896 \\ \mathrm{H} & 2.538234 & -2.358574 & -0.381102\end{array}$


$\mathrm{TS}\left(\mathbf{1} \rightarrow \mathbf{1 6}+\mathrm{H}_{2}\right)$ :

$$
\begin{aligned}
& E=-1215.35808477401 \mathrm{au} \\
& Z P E=0.06154899 \mathrm{au} \\
& \mathrm{G}_{\text {corr }}=0.03242271 \mathrm{au} \\
& v=-913.28 \mathrm{~cm}^{-1}
\end{aligned}
$$

$\begin{array}{lrrr}\mathrm{P} & -0.154209 & -0.120570 & 0.094961 \\ \mathrm{H} & 0.797157 & 1.135955 & -0.897086 \\ \mathrm{~S} & -1.688778 & 1.519251 & 0.092948 \\ \mathrm{C} & -1.033058 & -0.223220 & -1.631422 \\ \mathrm{H} & -0.463840 & -0.594213 & -2.482242\end{array}$

$\begin{array}{lrrr}\mathrm{H} & -1.887534 & -0.855889 & -1.401095 \\ \mathrm{C} & -1.327221 & 1.210871 & -1.559453 \\ \mathrm{H} & -1.513127 & 1.903543 & -2.367220 \\ \mathrm{H} & 0.272714 & 1.556848 & -1.494528 \\ \mathrm{~S} & -0.118115 & -1.576224 & 1.346113\end{array}$

16:

$$
\begin{aligned}
& E=-1214.23571268528 \mathrm{au} \\
& \mathrm{ZPE}=0.04637221 \mathrm{au} \\
& \mathrm{G}_{\text {corr }}=0.01551231 \mathrm{au}
\end{aligned}
$$

$\begin{array}{rrrr}\mathrm{P} & -0.477775 & 0.400904 & -0.298211 \\ \mathrm{~S} & 0.049163 & 0.339233 & 1.756259 \\ \mathrm{C} & 2.610055 & -0.128437 & 0.862197 \\ \mathrm{H} & 3.524485 & -0.707487 & 0.829976\end{array}$

$\mathrm{H} \quad 2.555600$

C 1.606929

H 1.684350

S $\quad-2.096408$

0.749972

$-0.496035$

$-1.363747$

1.429061

0.232313

1.651231

2.295875

$-0.450982$
$\mathrm{H}_{2}$ :

$\mathrm{TS}(\mathbf{1 6} \rightarrow \mathbf{1 7})$ :

17:

H $\quad 0.875063$

1.430295

$-1.034900$

H $\quad 0.448173$

1.780819

$-1.533445$

$$
\begin{aligned}
& E=-1.172380690754 \mathrm{au} \\
& Z P E=0.01006021 \mathrm{au} \\
& \mathrm{G}_{\text {corr }}=-0.00143069 \mathrm{au}
\end{aligned}
$$$$
\text { (n) }
$$ 


$\begin{array}{lrrrrrrr}\mathrm{H} & 2.286195 & 0.875755 & 0.799940 & \mathrm{H} & 1.169074 & -2.029909 & 0.986486 \\ \mathrm{C} & 0.960448 & -0.974499 & 0.924217 & \mathrm{~S} & -1.403037 & 0.301626 & -1.266568\end{array}$

\begin{tabular}{|c|c|c|c|c|c|c|}
\hline $\mathrm{TS}(\mathbf{1 7} \rightarrow \mathbf{1 8})$ & & $\begin{array}{l}\mathrm{E} \\
\mathrm{ZF} \\
\mathrm{G}_{\mathrm{c}} \\
\mathrm{v}=\end{array}$ & $37 \mathrm{a}$ & & & \\
\hline-0.009472 & -0.195060 & -0.325838 & $\mathrm{H}$ & 2.012887 & 1.011289 & 0.661530 \\
\hline-0.009646 & -0.204677 & 2.275379 & $\mathrm{C}$ & 0.943515 & -0.955627 & 1.060007 \\
\hline 1.667631 & 0.121433 & 0.143844 & $\mathrm{H}$ & 1.328059 & -1.967857 & 1.130063 \\
\hline 2.390709 & -0.319678 & -0.542871 & $\mathrm{~S}$ & -1.469797 & 0.743648 & -1.156313 \\
\hline
\end{tabular}

18:

$E=-1214.21285331886 \mathrm{au}$

$\mathrm{ZPE}=0.04651765 \mathrm{au}$

$\mathrm{G}_{\text {corr }}=0.01495357 \mathrm{au}$

$\begin{array}{rrrr}\mathrm{P} & -0.422813 & 0.654736 & -0.388398 \\ \mathrm{~S} & 0.625522 & -1.117739 & 2.694643 \\ \mathrm{C} & 1.265052 & 0.349981 & 0.450508 \\ \mathrm{H} & 2.022391 & 0.312911 & -0.332219\end{array}$

H 1.425092

1.175802

1.143011

$\begin{array}{llll}\text { C } & 1.108034 & -0.939456 & 1.157059\end{array}$

$\begin{array}{llll}\mathrm{H} & 1.261156 & -1.817705 & 0.530057\end{array}$

$\begin{array}{llll}\mathrm{H} & 2.022391 & 0.312911 & -0.332219\end{array}$

S $\quad-0.430548 \quad-0.385060$

$-2.008858$

$\mathrm{TS}(\mathbf{1 d} \rightarrow \mathbf{1 9}):$

$$
\begin{aligned}
& E=-1215.3501882163 \mathrm{au} \\
& Z P E=0.06599460 \mathrm{au} \\
& G_{\text {corr }}=0.03649397 \mathrm{au} \\
& v=-683.38 \mathrm{~cm}^{-1}
\end{aligned}
$$

$\begin{array}{lrrr}\mathrm{P} & 0.488800 & -0.064982 & -0.021700 \\ \mathrm{H} & 1.343257 & 0.282067 & 1.075586 \\ \mathrm{~S} & -0.036624 & 1.973994 & -0.500183 \\ \mathrm{C} & -0.969750 & -0.181699 & -1.859144 \\ \mathrm{H} & -0.146009 & -0.385730 & -2.529044\end{array}$

$\begin{array}{lrrr}\mathrm{H} & -1.771492 & -0.901541 & -1.898978 \\ \mathrm{C} & -1.318731 & 1.246252 & -1.598450 \\ \mathrm{H} & -2.284804 & 1.314696 & -1.103685 \\ \mathrm{H} & -1.335596 & 1.812219 & -2.535635 \\ \mathrm{~S} & -1.085061 & -1.138923 & 0.672212\end{array}$

19:

$$
\begin{aligned}
& E=-1215.46773203678 \mathrm{au} \\
& \mathrm{ZPE}=0.06903268 \mathrm{au} \\
& \mathrm{G}_{\text {corr }}=0.03978401 \mathrm{au}
\end{aligned}
$$

$\begin{array}{lrrr}\mathrm{P} & 0.940512 & 0.279898 & 0.416941 \\ \mathrm{H} & -0.107454 & 0.707198 & 1.281582 \\ \mathrm{~S} & 0.581160 & 1.714487 & -1.092757 \\ \mathrm{C} & -1.146696 & -0.353858 & -1.716404 \\ \mathrm{H} & -0.765383 & -0.562198 & -2.714332\end{array}$

$\begin{array}{lrrr}\mathrm{H} & -2.165554 & -0.735190 & -1.636041 \\ \mathrm{C} & -1.123952 & 1.140105 & -1.431330 \\ \mathrm{H} & -1.747042 & 1.385562 & -0.571284 \\ \mathrm{H} & -1.484507 & 1.699243 & -2.296899 \\ \mathrm{~S} & -0.097094 & -1.318895 & -0.538498\end{array}$




$$
\begin{aligned}
& \mathrm{ZPE}=0.14049971 \mathrm{au} \\
& \mathrm{G}_{\text {corr }}=0.10827138 \mathrm{au} \\
& v=-70.98 \mathrm{~cm}^{-1}
\end{aligned}
$$

$\begin{array}{rrrr}\mathrm{P} & -0.372796 & 0.125698 & -0.010526 \\ \mathrm{~S} & 3.366877 & 1.937214 & 0.765791 \\ \mathrm{C} & 0.729048 & 1.527992 & 0.194801 \\ \mathrm{H} & 0.339029 & 2.410508 & -0.311475 \\ \mathrm{H} & 0.780974 & 1.737524 & 1.267293 \\ \mathrm{C} & 2.157855 & 1.147886 & -0.297726 \\ \mathrm{H} & 2.230579 & 1.446720 & -1.350176 \\ \mathrm{H} & 2.271443 & 0.051279 & -0.290800 \\ \mathrm{H} & -1.729767 & 0.383817 & 0.263921\end{array}$

$\begin{array}{lrrr}\mathrm{C} & -0.353647 & -0.544841 & -1.685837 \\ \mathrm{H} & -0.987379 & -1.429308 & -1.750586 \\ \mathrm{H} & -0.713553 & 0.211755 & -2.382974 \\ \mathrm{H} & 0.670964 & -0.805543 & -1.947057 \\ \mathrm{C} & 0.095484 & -1.155240 & 1.170897 \\ \mathrm{H} & -0.452626 & -2.076687 & 0.977495 \\ \mathrm{H} & 1.167358 & -1.334385 & 1.094832 \\ \mathrm{H} & -0.125249 & -0.804104 & 2.178737\end{array}$

13e:

$$
\begin{aligned}
& E=-897.319427935186 \mathrm{au} \\
& \mathrm{ZPE}=0.14047430 \mathrm{au} \\
& \mathrm{G}_{\text {corr }}=0.10669259 \mathrm{au}
\end{aligned}
$$

$\begin{array}{rrrr}\mathrm{P} & -0.375385 & 0.116075 & 0.016859 \\ \mathrm{~S} & 3.297133 & 2.448550 & 0.293967 \\ \mathrm{C} & 0.777616 & 1.490731 & 0.265621 \\ \mathrm{H} & 0.386714 & 2.390510 & -0.210836 \\ \mathrm{H} & 0.844204 & 1.679786 & 1.339767 \\ \mathrm{C} & 2.187235 & 1.163050 & -0.284881 \\ \mathrm{H} & 2.126091 & 1.115842 & -1.379091 \\ \mathrm{H} & 2.489578 & 0.162762 & 0.060995 \\ \mathrm{H} & -1.732914 & 0.409828 & 0.253531\end{array}$

$\begin{array}{rrrr}\mathrm{C} & -0.315406 & -0.503314 & -1.676673 \\ \mathrm{H} & -1.006948 & -1.335504 & -1.806984 \\ \mathrm{H} & -0.575662 & 0.299672 & -2.366155 \\ \mathrm{H} & 0.701280 & -0.829455 & -1.892510 \\ \mathrm{C} & 0.002986 & -1.242784 & 1.143936 \\ \mathrm{H} & -0.665412 & -2.086441 & 0.973582 \\ \mathrm{H} & 1.035323 & -1.551978 & 0.983229 \\ \mathrm{H} & -0.101838 & -0.897047 & 2.172254\end{array}$

$\operatorname{TS}(\mathbf{1 3 e} \rightarrow \mathbf{1 1 + 1 2 e}):$

$$
\begin{aligned}
& E=-897.306764950491 \mathrm{au} \\
& \mathrm{ZPE}=0.13914041 \mathrm{au} \\
& \mathrm{G}_{\text {corr }}=0.10494962 \mathrm{au} \\
& v=-368.18 \mathrm{~cm}^{-1}
\end{aligned}
$$

$\begin{array}{rrrr}\text { P } & -0.535688 & 0.132209 & 0.076380 \\ \mathrm{~S} & 3.221432 & 2.586607 & 0.003740 \\ \mathrm{C} & 1.001990 & 1.697201 & 0.167665 \\ \mathrm{H} & 0.686393 & 2.270394 & -0.693546 \\ \mathrm{H} & 0.789337 & 2.170590 & 1.114698 \\ \mathrm{C} & 2.326019 & 1.019094 & 0.057537 \\ \mathrm{H} & 2.437372 & 0.421418 & -0.849110 \\ \mathrm{H} & 2.562622 & 0.411856 & 0.931484 \\ \mathrm{H} & -1.921517 & 0.093677 & 0.390013\end{array}$

$\begin{array}{rrrr}\mathrm{C} & -0.558130 & -0.375116 & -1.677658 \\ \mathrm{H} & -1.059148 & -1.332437 & -1.819902 \\ \mathrm{H} & -1.068394 & 0.387289 & -2.267092 \\ \mathrm{H} & 0.469177 & -0.450464 & -2.034418 \\ \mathrm{C} & 0.077833 & -1.332230 & 0.980216 \\ \mathrm{H} & -0.514208 & -2.219417 & 0.755715 \\ \mathrm{H} & 1.114744 & -1.510990 & 0.698257 \\ \mathrm{H} & 0.044758 & -1.139399 & 2.052633\end{array}$

\section{2e:}

$$
\begin{aligned}
& E=-421.177597951138 \mathrm{au} \\
& \mathrm{ZPE}=0.08349815 \mathrm{au} \\
& \mathrm{G}_{\text {corr }}=0.05734261 \mathrm{au} \\
& \mathrm{H}_{\text {corr }}=0.09393687 \mathrm{au}
\end{aligned}
$$

$\begin{array}{llll}\mathrm{P} & 0.001594 & 0.003074 & -0.016614\end{array}$

$\begin{array}{llll}\mathrm{H} & -0.010312 & -0.007697 & 1.403941\end{array}$ 


$\begin{array}{rrrrrrrr}\mathrm{C} & 1.845606 & -0.000168 & -0.226984 & \mathrm{C} & -0.346815 & 1.813301 & -0.231356 \\ \mathrm{H} & 2.342319 & 0.775573 & 0.357720 & \mathrm{H} & 0.316327 & 2.447446 & 0.358948 \\ \mathrm{H} & 2.241219 & -0.973972 & 0.065139 & \mathrm{H} & -0.233937 & 2.071660 & -1.285829 \\ \mathrm{H} & 2.080764 & 0.149598 & -1.282256 & \mathrm{H} & -1.380324 & 2.018676 & 0.051138\end{array}$

\begin{tabular}{|c|c|c|c|c|c|c|}
\hline 12c: & & $\begin{array}{l}\mathrm{E}= \\
\mathrm{ZP} \\
\mathrm{G}_{\mathrm{c}} \\
\mathrm{H}_{\mathrm{c}}\end{array}$ & $7 \mathrm{at}$ & & & \\
\hline-0.184945 & 0.884310 & 0.424445 & $\mathrm{O}$ & -1.173878 & -0.827331 & 1.663628 \\
\hline-1.152753 & -0.214393 & 0.312690 & & & & \\
\hline
\end{tabular}

TS-ii $(\mathbf{1 1}+\mathbf{1 2 c} \rightarrow \mathbf{4 + 5 c}): \quad E=-892.768280566444 \mathrm{au}$

$$
\begin{aligned}
& \mathrm{ZPE}=0.06624724 \mathrm{au} \\
& \mathrm{G}_{\text {corr }}=0.03573006 \mathrm{au} \\
& v=-386.10 \mathrm{~cm}^{-1}
\end{aligned}
$$

$\begin{array}{lrrrrrrr}\mathrm{S} & 0.015871 & -0.005155 & -0.003090 & \mathrm{H} & -0.160215 & 2.147135 & 1.879531 \\ \mathrm{C} & 0.009403 & -0.000757 & 2.057752 & \mathrm{H} & -1.754863 & 1.213351 & 1.785251 \\ \mathrm{H} & 1.052480 & 0.013364 & 2.343183 & \mathrm{P} & -1.004077 & -1.699700 & -0.704810 \\ \mathrm{H} & -0.518338 & -0.919336 & 2.288285 & \mathrm{H} & -2.336464 & -1.167371 & -0.766233 \\ \mathrm{C} & -0.680150 & 1.199668 & 1.888560 & \mathrm{O} & -0.967999 & -2.910130 & 0.169688\end{array}$

TS-i(11+12e $\rightarrow$ 4+5e): $\quad \quad E=-897.31606258646 \mathrm{au}$

$$
\begin{aligned}
& Z P E=0.13766971 \mathrm{au} \\
& \mathrm{G}_{\text {corr }}=0.10374898 \mathrm{au} \\
& v=-424.06 \mathrm{~cm}^{-1}
\end{aligned}
$$

$\begin{array}{rrrr}\text { S } & -0.010711 & -0.111906 & 0.005080 \\ \mathrm{C} & -0.021000 & 0.071811 & 2.186759 \\ \mathrm{H} & 1.019160 & 0.047765 & 2.476159 \\ \mathrm{H} & -0.639502 & -0.746090 & 2.525593 \\ \mathrm{C} & -0.584088 & 1.216613 & 1.654992 \\ \mathrm{H} & 0.004631 & 2.110832 & 1.513925\end{array}$

$\begin{array}{lrrr}\mathrm{H} & -1.655153 & 1.317912 & 1.561467 \\ \mathrm{P} & 0.789105 & -0.862253 & -2.225019 \\ \mathrm{H} & 0.241236 & -1.558638 & -3.338412 \\ \mathrm{C} & 1.468829 & 0.609493 & -3.086835 \\ \mathrm{H} & 2.121416 & 0.344864 & -3.920005 \\ \mathrm{H} & 0.644656 & 1.221743 & -3.453825\end{array}$




$\begin{array}{llllllll}\mathrm{H} & 2.026445 & 1.197268 & -2.357235 & \mathrm{H} & 2.870780 & -1.406139 & -1.144287 \\ \mathrm{C} & 2.277867 & -1.892601 & -1.919156 & \mathrm{H} & 1.966902 & -2.867958 & -1.544027 \\ \mathrm{H} & 2.886055 & -2.027228 & -2.814612 & & & & \end{array}$

12f:

$\begin{array}{lrrr}\mathrm{P} & -0.518746 & -2.167885 & -1.043032 \\ \mathrm{H} & -0.863031 & -3.319517 & -1.795310 \\ \mathrm{C} & -2.065347 & -2.047455 & -0.062890 \\ \mathrm{C} & -1.986740 & -1.552720 & 1.239785 \\ \mathrm{C} & -3.129323 & -1.434459 & 2.025481 \\ \mathrm{C} & -4.362726 & -1.820791 & 1.517850 \\ \mathrm{C} & -4.453528 & -2.317987 & 0.220240 \\ \mathrm{C} & -3.314577 & -2.425607 & -0.565085 \\ \mathrm{H} & -1.023406 & -1.262623 & 1.641528 \\ \mathrm{H} & -3.053022 & -1.047700 & 3.033830 \\ \mathrm{H} & -5.252223 & -1.737503 & 2.129431 \\ \mathrm{H} & -5.413862 & -2.620450 & -0.178229\end{array}$

$E=-803.919859451002 \mathrm{au}$

$\mathrm{ZPE}=0.19063988 \mathrm{au}$

$\mathrm{G}_{\text {corr }}=0.15640098 \mathrm{au}$

$\mathrm{H}_{\text {corr }}=0.20085504 \mathrm{au}$

\section{TS-i $(\mathbf{1 1}+\mathbf{1 2 f} \rightarrow \mathbf{4}+\mathbf{5 f}):$}

$\begin{array}{rrrr}\text { S } & -0.087166 & -0.091039 & -0.063837 \\ \mathrm{C} & 0.275275 & -0.058858 & 2.093893 \\ \mathrm{H} & 1.247607 & 0.398105 & 2.203398 \\ \mathrm{H} & 0.186682 & -1.090957 & 2.399197 \\ \mathrm{C} & -0.846482 & 0.706990 & 1.840183 \\ \mathrm{H} & -0.775681 & 1.780070 & 1.740883 \\ \mathrm{H} & -1.837982 & 0.289690 & 1.935731 \\ \mathrm{P} & 0.436223 & -0.364260 & -2.486349 \\ \mathrm{C} & 0.070343 & 1.174189 & -3.408862 \\ \mathrm{C} & -0.731024 & 2.143890 & -2.807469 \\ \mathrm{C} & -1.029700 & 3.323075 & -3.483032 \\ \mathrm{C} & -0.526912 & 3.541482 & -4.759263 \\ \mathrm{C} & 0.278037 & 2.579012 & -5.363224 \\ \mathrm{C} & 0.576502 & 1.402509 & -4.691243\end{array}$

$\begin{array}{lrrr}\mathrm{H} & -3.393150 & -2.799574 & -1.578171 \\ \mathrm{C} & -0.821852 & -0.950888 & -2.390157 \\ \mathrm{C} & -1.571144 & 0.208362 & -2.185439 \\ \mathrm{C} & -1.693955 & 1.158094 & -3.193940 \\ \mathrm{C} & -1.071431 & 0.963739 & -4.420621 \\ \mathrm{C} & -0.321412 & -0.188486 & -4.633706 \\ \mathrm{C} & -0.192978 & -1.133906 & -3.625311 \\ \mathrm{H} & -2.071433 & 0.366803 & -1.238854 \\ \mathrm{H} & -2.282514 & 2.050219 & -3.019487 \\ \mathrm{H} & -1.170092 & 1.701867 & -5.206138 \\ \mathrm{H} & 0.164022 & -0.351324 & -5.587898 \\ \mathrm{H} & 0.398010 & -2.025412 & -3.803061\end{array}$

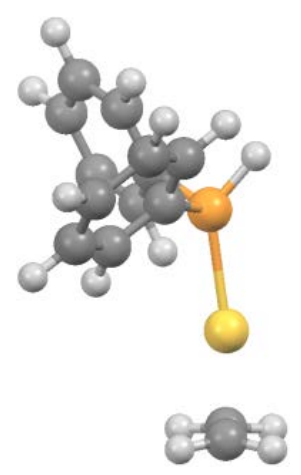

$$
\begin{aligned}
& E=-1280.05848487653 \mathrm{au} \\
& \mathrm{ZPE}=0.24470815 \mathrm{au} \\
& \mathrm{G}_{\text {corr }}=0.20545618 \mathrm{au} \\
& v=-418.77 \mathrm{~cm}^{-1}
\end{aligned}
$$




$\begin{array}{llll}\mathrm{H} & 4.599858 & -2.438509 & -4.226254 \\ \mathrm{H} & 2.152799 & -2.186232 & -4.077828\end{array}$

TS-ii $(\mathbf{1 1}+\mathbf{1 2 f} \rightarrow \mathbf{4 + 5 f}):$
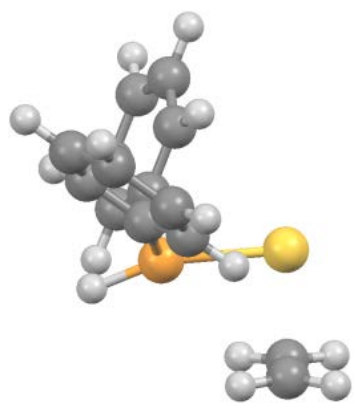

$\mathrm{E}=-1279.99258659943 \mathrm{au}$

$\mathrm{ZPE}=0.24407117 \mathrm{au}$

$\mathrm{G}_{\text {corr }}=0.20153357 \mathrm{au}$

$v=-608.34 \mathrm{~cm}^{-1}$

$\begin{array}{lrrr}\text { S } & 0.017368 & -0.045971 & 0.045746 \\ \mathrm{C} & -0.066315 & 0.048048 & 2.472050 \\ \mathrm{H} & 0.990844 & 0.049386 & 2.694288 \\ \mathrm{H} & -0.602028 & -0.866870 & 2.674791 \\ \mathrm{C} & -0.711853 & 1.182050 & 2.084123 \\ \mathrm{H} & -0.184891 & 2.117617 & 1.964425 \\ \mathrm{H} & -1.780695 & 1.197904 & 1.932733 \\ \mathrm{P} & -2.117021 & -1.183868 & 0.175006 \\ \mathrm{H} & -3.352988 & -1.752789 & 0.700526 \\ \mathrm{C} & -2.872727 & -0.432055 & -1.308577 \\ \mathrm{C} & -2.703725 & 0.918686 & -1.613212 \\ \mathrm{C} & -3.357230 & 1.480614 & -2.702926 \\ \mathrm{C} & -4.180014 & 0.697683 & -3.505603 \\ \mathrm{C} & -4.351530 & -0.649921 & -3.211291 \\ \mathrm{C} & -3.703607 & -1.211718 & -2.117639 \\ \mathrm{H} & -2.041959 & 1.521351 & -1.007395\end{array}$

$\begin{array}{lrrr}\mathrm{H} & -3.218058 & 2.530347 & -2.928625 \\ \mathrm{H} & -4.682356 & 1.135339 & -4.359062 \\ \mathrm{H} & -4.987843 & -1.266404 & -3.833700 \\ \mathrm{H} & -3.836129 & -2.262799 & -1.895891 \\ \mathrm{C} & -1.444974 & -2.782782 & -0.341032 \\ \mathrm{C} & -0.746163 & -2.928187 & -1.542013 \\ \mathrm{C} & -0.164424 & -4.147129 & -1.870203 \\ \mathrm{C} & -0.268223 & -5.227582 & -1.002351 \\ \mathrm{C} & -0.966421 & -5.091497 & 0.194730 \\ \mathrm{C} & -1.556047 & -3.878318 & 0.520018 \\ \mathrm{H} & -0.673345 & -2.089381 & -2.220218 \\ \mathrm{H} & 0.369867 & -4.251816 & -2.806024 \\ \mathrm{H} & 0.186035 & -6.176118 & -1.259247 \\ \mathrm{H} & -1.053158 & -5.932149 & 0.871399 \\ \mathrm{H} & -2.106463 & -3.778919 & 1.448321\end{array}$

$5 f:$

$\begin{array}{lrrr}\text { S } & -3.778979 & 2.000599 & -0.463632 \\ \text { P } & -3.216371 & 0.313075 & -1.243842 \\ \text { H } & -4.139301 & -0.289911 & -2.126930 \\ \mathrm{C} & -2.944714 & -1.055545 & -0.086225 \\ \mathrm{C} & -2.165153 & -0.845961 & 1.052019 \\ \mathrm{C} & -1.936680 & -1.889459 & 1.936515 \\ \mathrm{C} & -2.491711 & -3.143937 & 1.696059 \\ \mathrm{C} & -3.276079 & -3.353749 & 0.568910 \\ \mathrm{C} & -3.501938 & -2.310685 & -0.323014 \\ \mathrm{H} & -1.756763 & 0.137921 & 1.245679 \\ \mathrm{H} & -1.331419 & -1.724650 & 2.818653 \\ \mathrm{H} & -2.318274 & -3.954501 & 2.392243 \\ \mathrm{H} & -3.717330 & -4.325148 & 0.386350\end{array}$

$$
\begin{aligned}
& E=-1201.68477291476 \mathrm{au} \\
& \mathrm{ZPE}=0.193936108 \mathrm{au} \\
& \mathrm{G}_{\text {corr }}=0.15773917 \mathrm{au} \\
& \mathrm{H}_{\text {corr }}=0.20538869 \mathrm{au}
\end{aligned}
$$




$$
\mathrm{E}=-460.426147479334 \mathrm{au}
$$

$$
\mathrm{ZPE}=0.11213011 \mathrm{au}
$$$$
\mathrm{G}_{\text {corr }}=0.08393214 \mathrm{au}
$$$$
\mathrm{H}_{\text {corr }}=0.11974680 \mathrm{au}
$$

$\begin{array}{lllr}\mathrm{P} & -1.188682 & -1.457088 & -1.484512 \\ \mathrm{C} & -2.612178 & -1.857395 & -0.371356 \\ \mathrm{H} & -3.434814 & -2.338098 & -0.907906 \\ \mathrm{H} & -2.275423 & -2.521627 & 0.426402 \\ \mathrm{H} & -2.981965 & -0.941268 & 0.092309 \\ \mathrm{C} & -2.104002 & -0.587501 & -2.837410 \\ \mathrm{H} & -2.959666 & -1.166386 & -3.195611\end{array}$

$\begin{array}{lrrr}\mathrm{H} & -2.458775 & 0.378966 & -2.475253 \\ \mathrm{H} & -1.427957 & -0.402150 & -3.673810 \\ \mathrm{C} & -0.968581 & -3.109254 & -2.288944 \\ \mathrm{H} & -0.239881 & -3.028566 & -3.097159 \\ \mathrm{H} & -0.578887 & -3.820584 & -1.558750 \\ \mathrm{H} & -1.905905 & -3.498829 & -2.695633\end{array}$

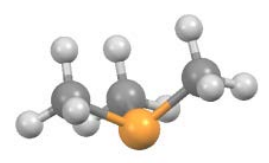

TS-i (11+20a $\rightarrow$ 4+21a): $\quad E=-936.567154193583$ au

$$
\begin{aligned}
& Z P E=0.16604395 \mathrm{au} \\
& G_{\text {corr }}=0.13133016 \mathrm{au} \\
& v=-393.41 \mathrm{~cm}^{-1}
\end{aligned}
$$

$\begin{array}{lrrr}\mathrm{S} & 0.440010 & -0.125333 & -0.037694 \\ \mathrm{C} & 1.297664 & 1.707786 & 0.778633 \\ \mathrm{H} & 0.505421 & 2.440127 & 0.733157 \\ \mathrm{H} & 2.176237 & 1.900830 & 0.181155 \\ \mathrm{C} & 1.301425 & 0.722359 & 1.752610 \\ \mathrm{H} & 0.520813 & 0.678678 & 2.497737 \\ \mathrm{H} & 2.189838 & 0.139882 & 1.947299 \\ \mathrm{P} & -0.748522 & -1.909761 & -1.404903 \\ \mathrm{C} & -1.088591 & -3.279532 & -0.233543 \\ \mathrm{H} & -1.612716 & -2.878999 & 0.634413\end{array}$

$\begin{array}{lrrr}\mathrm{H} & -1.689592 & -4.071191 & -0.687463 \\ \mathrm{H} & -0.141788 & -3.698124 & 0.109371 \\ \mathrm{C} & -0.047349 & -2.808865 & -2.849171 \\ \mathrm{H} & 0.106253 & -2.107601 & -3.670305 \\ \mathrm{H} & 0.923451 & -3.222528 & -2.573492 \\ \mathrm{H} & -0.699003 & -3.618807 & -3.187709 \\ \mathrm{C} & -2.433147 & -1.484553 & -1.999893 \\ \mathrm{H} & -2.357512 & -0.747303 & -2.800077 \\ \mathrm{H} & -2.974221 & -2.359510 & -2.369235 \\ \mathrm{H} & -2.991825 & -1.034232 & -1.178965\end{array}$

\section{1a:}

$$
\begin{aligned}
& E=-858.201160103921 \mathrm{au} \\
& Z P E=0.11529234 \mathrm{au} \\
& G_{\text {corr }}=0.08483467 \mathrm{au} \\
& H_{\text {corr }}=0.12409840 \mathrm{au}
\end{aligned}
$$

$\begin{array}{lrrr}\mathrm{S} & 0.262424 & -0.656651 & -0.763837 \\ \mathrm{P} & -1.300627 & -1.522038 & -1.538547 \\ \mathrm{C} & -2.636669 & -1.861342 & -0.347968 \\ \mathrm{H} & -3.489838 & -2.342020 & -0.830322 \\ \mathrm{H} & -2.247853 & -2.506153 & 0.439655 \\ \mathrm{H} & -2.951212 & -0.919551 & 0.100899 \\ \mathrm{C} & -2.115469 & -0.569522 & -2.859975\end{array}$

$\begin{array}{lrrr}\mathrm{H} & -2.978631 & -1.103539 & -3.261761 \\ \mathrm{H} & -2.434131 & 0.390460 & -2.454533 \\ \mathrm{H} & -1.393601 & -0.386155 & -3.655305 \\ \mathrm{C} & -0.960223 & -3.139785 & -2.302756 \\ \mathrm{H} & -0.219170 & -3.005070 & -3.090133 \\ \mathrm{H} & -0.543119 & -3.802964 & -1.545488 \\ \mathrm{H} & -1.866174 & -3.582101 & -2.721396\end{array}$


20b:

$$
\begin{aligned}
& E=-1034.5409118531 \mathrm{au} \\
& \mathrm{ZPE}=0.27305441 \mathrm{au} \\
& \mathrm{G}_{\text {corr }}=0.23204781 \mathrm{au} \\
& \mathrm{H}_{\text {corr }}=0.28808520 \mathrm{au}
\end{aligned}
$$

$\begin{array}{lrrr}\mathrm{P} & 0.009264 & -0.077873 & -0.212586 \\ \mathrm{C} & 1.831831 & -0.007374 & 0.012799 \\ \mathrm{C} & 2.630545 & 1.072024 & -0.368414 \\ \mathrm{C} & 4.004621 & 1.043571 & -0.156009 \\ \mathrm{C} & 4.600327 & -0.066155 & 0.431792 \\ \mathrm{C} & 3.813747 & -1.148043 & 0.814310 \\ \mathrm{C} & 2.440366 & -1.112899 & 0.615330 \\ \mathrm{H} & 2.178402 & 1.937974 & -0.832730 \\ \mathrm{H} & 4.610547 & 1.890278 & -0.453938 \\ \mathrm{H} & 5.670290 & -0.087282 & 0.595534 \\ \mathrm{H} & 4.269234 & -2.014386 & 1.277309 \\ \mathrm{H} & 1.829614 & -1.951329 & 0.929632 \\ \mathrm{C} & -0.374324 & 1.656193 & -0.685415 \\ \mathrm{C} & -0.581383 & 2.083810 & -1.997474 \\ \mathrm{C} & -0.886461 & 3.413829 & -2.267958 \\ \mathrm{C} & -0.979020 & 4.336497 & -1.233521 \\ \mathrm{C} & -0.774078 & 3.920977 & 0.078893\end{array}$

$\begin{array}{lrrr}\mathrm{C} & -0.486212 & 2.590707 & 0.349800 \\ \mathrm{H} & -0.503982 & 1.377837 & -2.812739 \\ \mathrm{H} & -1.047300 & 3.728159 & -3.291733 \\ \mathrm{H} & -1.214805 & 5.371554 & -1.445841 \\ \mathrm{H} & -0.849171 & 4.632002 & 0.892064 \\ \mathrm{H} & -0.343459 & 2.270562 & 1.375477 \\ \mathrm{C} & -0.131735 & -0.941627 & -1.828005 \\ \mathrm{C} & -1.381442 & -1.472235 & -2.160628 \\ \mathrm{C} & -1.582688 & -2.107306 & -3.379316 \\ \mathrm{C} & -0.528966 & -2.237324 & -4.277747 \\ \mathrm{C} & 0.722613 & -1.727900 & -3.950121 \\ \mathrm{C} & 0.920079 & -1.081925 & -2.735163 \\ \mathrm{H} & -2.201189 & -1.384262 & -1.457182 \\ \mathrm{H} & -2.558431 & -2.508113 & -3.623603 \\ \mathrm{H} & -0.680997 & -2.738914 & -5.225049 \\ \mathrm{H} & 1.547955 & -1.829329 & -4.643893 \\ \mathrm{H} & 1.895932 & -0.683425 & -2.492420\end{array}$

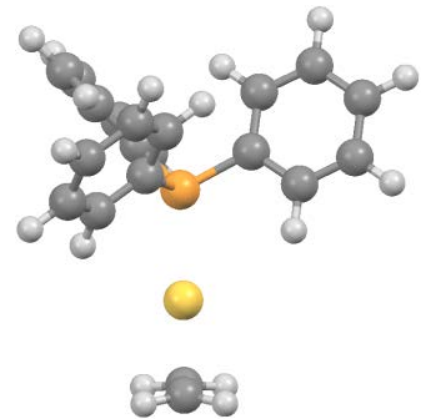

$\mathrm{TS}-\mathrm{i}(\mathbf{1 1}+\mathbf{2 0 b} \rightarrow \mathbf{4}+\mathbf{2 1 b})$

$$
\begin{aligned}
& E=-1510.68340295117 \mathrm{au} \\
& \mathrm{ZPE}=0.32693591 \mathrm{au} \\
& \mathrm{G}_{\mathrm{corr}}=0.28275798 \mathrm{au} \\
& v=-391.54 \mathrm{~cm}^{-1}
\end{aligned}
$$

$\begin{array}{lrrr}\mathrm{S} & 0.041052 & -0.023818 & 0.021903 \\ \mathrm{C} & 0.484650 & 0.019259 & 2.142674 \\ \mathrm{H} & 1.434597 & 0.526262 & 2.225036 \\ \mathrm{H} & 0.455820 & -1.011400 & 2.463745 \\ \mathrm{C} & -0.685092 & 0.729803 & 1.927325 \\ \mathrm{H} & -0.671504 & 1.805240 & 1.829954 \\ \mathrm{H} & -1.649682 & 0.265733 & 2.070575 \\ \mathrm{P} & 0.310393 & -0.514712 & -2.453497 \\ \mathrm{C} & -0.251566 & 0.791657 & -3.593262 \\ \mathrm{C} & -1.524137 & 1.329089 & -3.373596 \\ \mathrm{C} & -2.022737 & 2.319665 & -4.207172 \\ \mathrm{C} & -1.249025 & 2.800599 & -5.259727 \\ \mathrm{C} & 0.023397 & 2.284623 & -5.473106 \\ \mathrm{C} & 0.520926 & 1.283718 & -4.645770 \\ \mathrm{H} & -2.118685 & 0.969976 & -2.542240 \\ \mathrm{H} & -3.011383 & 2.724155 & -4.030701\end{array}$

$\begin{array}{rrrr}\mathrm{H} & -1.635095 & 3.578259 & -5.906410 \\ \mathrm{H} & 0.631395 & 2.658198 & -6.287542 \\ \mathrm{H} & 1.510517 & 0.883992 & -4.820582 \\ \mathrm{C} & 2.067532 & -0.752766 & -2.875573 \\ \mathrm{C} & 2.999193 & 0.066911 & -2.230999 \\ \mathrm{C} & 4.352704 & -0.050230 & -2.516910 \\ \mathrm{C} & 4.792915 & -0.997388 & -3.436304 \\ \mathrm{C} & 3.873563 & -1.826640 & -4.068528 \\ \mathrm{C} & 2.516795 & -1.704893 & -3.791999 \\ \mathrm{H} & 2.654110 & 0.784758 & -1.497314 \\ \mathrm{H} & 5.065791 & 0.591118 & -2.014435 \\ \mathrm{H} & 5.849165 & -1.093226 & -3.653785 \\ \mathrm{H} & 4.211535 & -2.569922 & -4.779710 \\ \mathrm{H} & 1.806856 & -2.352528 & -4.288380 \\ \mathrm{C} & -0.508397 & -2.015910 & -3.087781 \\ \mathrm{C} & -0.642814 & -3.093157 & -2.207993\end{array}$




$\begin{array}{llllllll}\mathrm{C} & -1.232737 & -4.276509 & -2.632775 & \mathrm{H} & -1.331603 & -5.106099 & -1.944101 \\ \mathrm{C} & -1.709150 & -4.390244 & -3.934915 & \mathrm{H} & -2.176493 & -5.309944 & -4.263492 \\ \mathrm{C} & -1.593658 & -3.316345 & -4.810992 & \mathrm{H} & -1.969353 & -3.398298 & -5.823246 \\ \mathrm{C} & -0.995685 & -2.134326 & -4.391047 & \mathrm{H} & -0.908376 & -1.301453 & -5.076071 \\ \mathrm{H} & -0.296607 & -2.988249 & -1.186964 & & & & \end{array}$

\section{1b:}

$$
\begin{aligned}
& E=-1432.31090221367 \mathrm{au} \\
& \mathrm{ZPE}=0.27607194 \mathrm{au} \\
& \mathrm{G}_{\text {corr }}=0.23347470 \mathrm{au} \\
& \mathrm{H}_{\text {corr }}=0.29243769 \mathrm{au}
\end{aligned}
$$

$\begin{array}{lrrr}\mathrm{S} & 0.859295 & -1.961972 & -0.068227 \\ \mathrm{P} & -0.683250 & -1.981957 & -1.263605 \\ \mathrm{C} & -2.283444 & -1.726990 & -0.426505 \\ \mathrm{C} & -2.310846 & -1.017422 & 0.773562 \\ \mathrm{C} & -3.520657 & -0.771972 & 1.410996 \\ \mathrm{C} & -4.708556 & -1.238049 & 0.858333 \\ \mathrm{C} & -4.685172 & -1.954382 & -0.333425 \\ \mathrm{C} & -3.477568 & -2.199167 & -0.974746 \\ \mathrm{H} & -1.378276 & -0.675941 & 1.203816 \\ \mathrm{H} & -3.534844 & -0.221643 & 2.343098 \\ \mathrm{H} & -5.650163 & -1.050407 & 1.358640 \\ \mathrm{H} & -5.606337 & -2.328702 & -0.761729 \\ \mathrm{H} & -3.466450 & -2.765039 & -1.896252 \\ \mathrm{C} & -0.617400 & -0.705404 & -2.560409 \\ \mathrm{C} & -1.631757 & 0.231805 & -2.744459 \\ \mathrm{C} & -1.516581 & 1.198794 & -3.738538 \\ \mathrm{C} & -0.395555 & 1.227414 & -4.557135 \\ \mathrm{C} & 0.620476 & 0.291819 & -4.376378\end{array}$

$\begin{array}{lrrr}\mathrm{C} & 0.514572 & -0.665036 & -3.379222 \\ \mathrm{H} & -2.508657 & 0.213720 & -2.112560 \\ \mathrm{H} & -2.305069 & 1.928819 & -3.869666 \\ \mathrm{H} & -0.307529 & 1.979503 & -5.330803 \\ \mathrm{H} & 1.499075 & 0.314636 & -5.008271 \\ \mathrm{H} & 1.313433 & -1.378214 & -3.220998 \\ \mathrm{C} & -0.885149 & -3.544201 & -2.180836 \\ \mathrm{C} & -0.527634 & -4.737093 & -1.551718 \\ \mathrm{C} & -0.698773 & -5.948740 & -2.206707 \\ \mathrm{C} & -1.221004 & -5.978517 & -3.496029 \\ \mathrm{C} & -1.572328 & -4.792453 & -4.129777 \\ \mathrm{C} & -1.406621 & -3.577554 & -3.474800 \\ \mathrm{H} & -0.098696 & -4.701515 & -0.558606 \\ \mathrm{H} & -0.416439 & -6.870360 & -1.713944 \\ \mathrm{H} & -1.345989 & -6.924022 & -4.008304 \\ \mathrm{H} & -1.969489 & -4.810453 & -5.136707 \\ \mathrm{H} & -1.671176 & -2.656567 & -3.976786\end{array}$

20c:

$$
\begin{aligned}
& E=-685.84629038455 \mathrm{au} \\
& Z P E=0.12792222 \mathrm{au} \\
& \mathrm{G}_{\text {corr }}=0.09226923 \mathrm{au} \\
& \mathrm{H}_{\text {corr }}=0.13917022 \mathrm{au}
\end{aligned}
$$

$\begin{array}{rrrr}\mathrm{P} & -0.017560 & 0.026151 & -0.021919 \\ \mathrm{O} & 1.613872 & -0.002414 & -0.054346 \\ \mathrm{C} & 2.315104 & 1.239393 & -0.000529 \\ \mathrm{H} & 3.307156 & 1.070336 & -0.415918 \\ \mathrm{H} & 1.806704 & 2.010990 & -0.587508 \\ \mathrm{H} & 2.407226 & 1.579125 & 1.033078 \\ \mathrm{O} & -0.280034 & -1.572934 & -0.217183 \\ \mathrm{C} & -0.295208 & -2.099185 & -1.543783\end{array}$

$\begin{array}{rrrr}\mathrm{H} & -0.850980 & -3.034926 & -1.515703 \\ \mathrm{H} & -0.784808 & -1.411459 & -2.240701 \\ \mathrm{H} & 0.723083 & -2.291672 & -1.887716 \\ \mathrm{O} & -0.214682 & 0.095853 & 1.596667 \\ \mathrm{C} & -1.539321 & 0.220271 & 2.113036 \\ \mathrm{H} & -1.458637 & 0.630408 & 3.118283 \\ \mathrm{H} & -2.146104 & 0.892826 & 1.498336 \\ \mathrm{H} & -2.023155 & -0.757571 & 2.157439\end{array}$


$\mathrm{TS}-\mathrm{i}(11+20 \mathrm{c} \rightarrow \mathbf{4 + 2 1 c})$ :
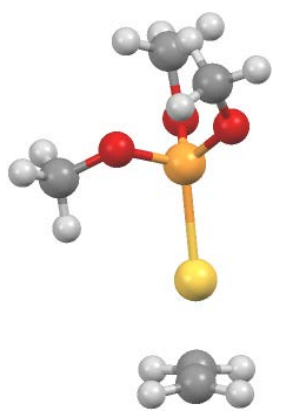

$\mathrm{E}=-1161.98494424454 \mathrm{au}$

$\mathrm{ZPE}=0.18228136 \mathrm{au}$

$\mathrm{G}_{\text {corr }}=0.14193744 \mathrm{au}$

$v=-406.92 \mathrm{~cm}^{-1}$

$\begin{array}{lrrr}\mathrm{S} & 0.028820 & -0.009902 & -0.000619 \\ \mathrm{C} & -0.022538 & 0.008016 & 2.185230 \\ \mathrm{H} & 1.015630 & 0.031762 & 2.481787 \\ \mathrm{H} & -0.586216 & -0.871932 & 2.458063 \\ \mathrm{C} & -0.657763 & 1.146280 & 1.726606 \\ \mathrm{H} & -0.130080 & 2.085636 & 1.652650 \\ \mathrm{H} & -1.732809 & 1.183143 & 1.630265 \\ \mathrm{P} & 0.260986 & -0.788657 & -2.366678 \\ \mathrm{O} & 0.910195 & 0.104995 & -3.540282 \\ \mathrm{C} & 0.391120 & 0.306378 & -4.861955 \\ \mathrm{H} & 1.066808 & 1.006272 & -5.349421 \\ \mathrm{H} & 0.363992 & -0.628333 & -5.422984\end{array}$

$\begin{array}{llll}\mathrm{H} & -0.612672 & 0.726263 & -4.818969\end{array}$

$\begin{array}{llll}\mathrm{O} & 0.977549 & -2.233860 & -2.487843\end{array}$

$\begin{array}{llll}\text { C } & 1.114379 & -2.933622 & -3.730656\end{array}$

$\mathrm{H} \quad 1.833520 \quad-2.422966 \quad-4.373160$

$\begin{array}{llll}\mathrm{H} & 1.482878 & -3.930078 & -3.495848\end{array}$

$\begin{array}{llll}\mathrm{H} & 0.153127 & -3.009967 & -4.242310\end{array}$

$\begin{array}{llll}\text { O } & -1.201014 & -1.032418 & -3.050816\end{array}$

$\begin{array}{llll}\text { C } & -2.208479 & -1.730678 & -2.306880\end{array}$

$\mathrm{H} \quad-2.262291 \quad-1.349446 \quad-1.286231$

$\begin{array}{llll}\mathrm{H} & -3.152183 & -1.562499 & -2.822007\end{array}$

$\begin{array}{llll}\mathrm{H} & -1.990552 & -2.800657 & -2.283305\end{array}$

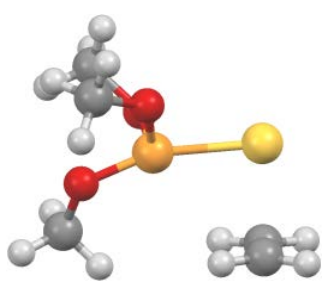

TS-ii(11+20c $\rightarrow$ 4+21c): $\quad E=-1161.91483055104 \mathrm{au}$

$$
\mathrm{ZPE}=0.18176765 \mathrm{au}
$$

$\mathrm{G}_{\text {corr }}=0.14139435 \mathrm{au}$

$v=-553.92 \mathrm{~cm}^{-1}$

$\begin{array}{lrrr}\mathrm{S} & 0.045821 & -0.041771 & 0.028771 \\ \mathrm{C} & -0.011927 & 0.029945 & 2.450981 \\ \mathrm{H} & 1.045652 & 0.027333 & 2.671829 \\ \mathrm{H} & -0.548116 & -0.885349 & 2.648714 \\ \mathrm{C} & -0.654120 & 1.167768 & 2.062836 \\ \mathrm{H} & -0.124788 & 2.102547 & 1.946677 \\ \mathrm{H} & -1.722368 & 1.188816 & 1.910497 \\ \mathrm{P} & -2.045114 & -1.127209 & 0.164014 \\ \mathrm{O} & -2.442314 & -0.658879 & -1.328159 \\ \mathrm{C} & -3.756032 & -0.667112 & -1.889362 \\ \mathrm{H} & -3.652731 & -0.257338 & -2.892749 \\ \mathrm{H} & -4.158166 & -1.678846 & -1.944175\end{array}$

$\begin{array}{llrr}\mathrm{H} & -4.437490 & -0.049916 & -1.304920 \\ \mathrm{O} & -1.467046 & -2.636763 & 0.017308 \\ \mathrm{C} & -2.096312 & -3.597668 & -0.830706 \\ \mathrm{H} & -2.010083 & -3.297227 & -1.876562 \\ \mathrm{H} & -1.571407 & -4.539211 & -0.681473 \\ \mathrm{H} & -3.150553 & -3.721225 & -0.572156 \\ \mathrm{O} & -3.634470 & -1.470106 & 0.631461 \\ \mathrm{C} & -3.837845 & -1.933413 & 1.957293 \\ \mathrm{H} & -3.306522 & -1.309746 & 2.685264 \\ \mathrm{H} & -4.906441 & -1.882342 & 2.162665 \\ \mathrm{H} & -3.498136 & -2.967086 & 2.071479\end{array}$


$\mathrm{E}=-1083.63461184043 \mathrm{au}$

$\mathrm{ZPE}=0.13208154 \mathrm{au}$

$\mathrm{G}_{\text {corr }}=0.09524582 \mathrm{au}$

$\mathrm{H}_{\text {corr }}=0.14420393 \mathrm{au}$

$\begin{array}{rrrr}\mathrm{P} & 0.030366 & -0.046114 & 0.041607 \\ \mathrm{O} & 1.617172 & 0.018775 & -0.004567 \\ \mathrm{C} & 2.308124 & 1.277532 & -0.022457 \\ \mathrm{H} & 3.354719 & 1.047240 & -0.205169 \\ \mathrm{H} & 1.914278 & 1.912226 & -0.816235 \\ \mathrm{H} & 2.200500 & 1.776891 & 0.941156 \\ \mathrm{O} & -0.225612 & -1.603964 & -0.136922 \\ \mathrm{C} & -0.227176 & -2.210398 & -1.438779 \\ \mathrm{H} & -0.579195 & -3.229388 & -1.299237\end{array}$

$\begin{array}{lrrr}\mathrm{H} & -0.894236 & -1.666045 & -2.107225 \\ \mathrm{H} & 0.783735 & -2.219331 & -1.848019 \\ \mathrm{O} & -0.248376 & 0.144098 & 1.594155 \\ \mathrm{C} & -1.584763 & 0.312155 & 2.092547 \\ \mathrm{H} & -1.486744 & 0.570189 & 3.143997 \\ \mathrm{H} & -2.091106 & 1.110698 & 1.550405 \\ \mathrm{H} & -2.142577 & -0.619271 & 1.988929 \\ \mathrm{~S} & -0.918827 & 1.127127 & -1.154504\end{array}$

20d:

$$
\begin{aligned}
& E=-743.939051189062 \mathrm{au} \\
& \mathrm{ZPE}=0.24841702 \mathrm{au} \\
& \mathrm{G}_{\text {corr }}=0.20897133 \mathrm{au} \\
& \mathrm{H}_{\text {corr }}=0.26389810 \mathrm{au}
\end{aligned}
$$

$\begin{array}{lrrr}\mathrm{P} & -1.865238 & -1.293306 & -0.787637 \\ \mathrm{~N} & -1.925747 & -0.272811 & -2.201083 \\ \mathrm{C} & -2.953587 & 0.761904 & -2.158499 \\ \mathrm{H} & -2.715724 & 1.565655 & -1.444234 \\ \mathrm{H} & -3.049933 & 1.213440 & -3.149214 \\ \mathrm{H} & -3.912952 & 0.334406 & -1.878698 \\ \mathrm{C} & -0.641855 & 0.294737 & -2.598975 \\ \mathrm{H} & -0.281554 & 1.062122 & -1.895989 \\ \mathrm{H} & 0.113824 & -0.483563 & -2.667293 \\ \mathrm{H} & -0.747026 & 0.764908 & -3.580059 \\ \mathrm{~N} & -0.755850 & -2.440464 & -1.381331 \\ \mathrm{C} & -0.836621 & -3.086123 & -2.677665 \\ \mathrm{H} & -1.394815 & -2.459580 & -3.369650 \\ \mathrm{H} & 0.167966 & -3.238762 & -3.088129\end{array}$

$\begin{array}{lrrr}\mathrm{H} & -1.324536 & -4.068621 & -2.619237 \\ \mathrm{C} & 0.052696 & -3.156723 & -0.414506 \\ \mathrm{H} & 0.057533 & -2.617789 & 0.533035 \\ \mathrm{H} & -0.323099 & -4.172971 & -0.229182 \\ \mathrm{H} & 1.086900 & -3.244487 & -0.766943 \\ \mathrm{~N} & -3.451440 & -1.903702 & -0.882456 \\ \mathrm{C} & -4.082204 & -2.334488 & 0.349564 \\ \mathrm{H} & -3.558169 & -1.902483 & 1.202323 \\ \mathrm{H} & -5.127040 & -2.004393 & 0.383126 \\ \mathrm{H} & -4.075880 & -3.428037 & 0.460945 \\ \mathrm{C} & -4.084809 & -2.415173 & -2.082707 \\ \mathrm{H} & -4.030583 & -3.510623 & -2.143109 \\ \mathrm{H} & -5.144181 & -2.134346 & -2.100522 \\ \mathrm{H} & -3.606691 & -1.992468 & -2.963364\end{array}$

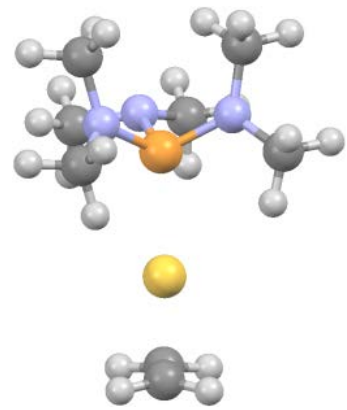

TS-i(11+20d $\rightarrow$ 4+21d): $\quad E=-1220.08379155906 \mathrm{au}$

$$
\begin{aligned}
& Z P E=0.30258982 \mathrm{au} \\
& G_{\text {corr }}=0.25939162 \mathrm{au} \\
& v=-381.16 \mathrm{~cm}^{-1}
\end{aligned}
$$

$\begin{array}{llll}\mathrm{S} & 0.000605 & 0.001519 & 0.005976 \\ \mathrm{C} & 0.178003 & 0.018652 & 2.156105 \\ \mathrm{H} & 1.221857 & 0.201406 & 2.363850\end{array}$

$\begin{array}{lrrr}\mathrm{H} & -0.226043 & -0.924801 & 2.492045 \\ \mathrm{C} & -0.660710 & 1.052052 & 1.761447 \\ \mathrm{H} & -0.286373 & 2.060080 & 1.661013\end{array}$




$\begin{array}{lrrr}\mathrm{H} & -1.733891 & 0.934310 & 1.788391 \\ \mathrm{P} & 0.254005 & -0.477110 & -2.524453 \\ \mathrm{~N} & -0.301725 & 0.835159 & -3.487939 \\ \mathrm{C} & -1.739232 & 1.088705 & -3.439406 \\ \mathrm{H} & -2.044663 & 1.569523 & -2.499932 \\ \mathrm{H} & -2.012705 & 1.749976 & -4.265472 \\ \mathrm{H} & -2.292048 & 0.158595 & -3.540939 \\ \mathrm{C} & 0.454035 & 2.079177 & -3.361681 \\ \mathrm{H} & 0.239683 & 2.599771 & -2.418666 \\ \mathrm{H} & 1.521338 & 1.880239 & -3.404549 \\ \mathrm{H} & 0.187846 & 2.743046 & -4.188123 \\ \mathrm{~N} & 1.847972 & -0.564549 & -3.071507 \\ \mathrm{C} & 2.239444 & -0.586817 & -4.469320 \\ \mathrm{H} & 1.475347 & -0.107938 & -5.077746 \\ \mathrm{H} & 3.177040 & -0.036678 & -4.603746\end{array}$

$\begin{array}{lrrr}\mathrm{H} & 2.395058 & -1.609037 & -4.838312 \\ \mathrm{C} & 2.852735 & -1.073588 & -2.154107 \\ \mathrm{H} & 2.494727 & -0.979767 & -1.129632 \\ \mathrm{H} & 3.093993 & -2.127245 & -2.348627 \\ \mathrm{H} & 3.777133 & -0.494114 & -2.250497 \\ \mathrm{~N} & -0.697374 & -1.717244 & -3.159090 \\ \mathrm{C} & -1.112372 & -2.792814 & -2.275611 \\ \mathrm{H} & -1.001201 & -2.477363 & -1.238977 \\ \mathrm{H} & -2.164859 & -3.040634 & -2.452492 \\ \mathrm{H} & -0.521493 & -3.704984 & -2.432193 \\ \mathrm{C} & -0.850317 & -1.999733 & -4.574522 \\ \mathrm{H} & -0.196649 & -2.817964 & -4.903242 \\ \mathrm{H} & -1.884232 & -2.290857 & -4.790400 \\ \mathrm{H} & -0.620563 & -1.111571 & -5.158957\end{array}$

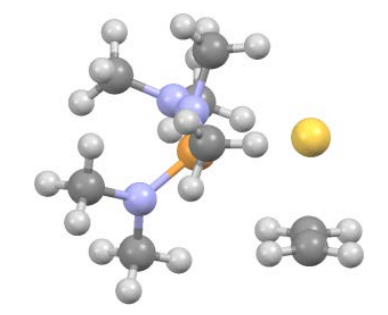

$\begin{array}{ll}\text { TS-ii }(\mathbf{1 1 + 2 0 d} \rightarrow \mathbf{4 + 2 1 d}): & \mathrm{E}=-1220.00797317276 \mathrm{au} \\ & \mathrm{ZPE}=0.30224039 \mathrm{au} \\ & \mathrm{G}_{\mathrm{corr}}=0.25561046 \mathrm{au} \\ & v=-494.98 \mathrm{~cm}^{-1}\end{array}$

$\begin{array}{lrrr}\mathrm{S} & 0.012251 & -0.018718 & 0.004079 \\ \mathrm{C} & -0.007629 & -0.001439 & 2.476553 \\ \mathrm{H} & 1.056470 & -0.020060 & 2.660735 \\ \mathrm{H} & -0.545513 & -0.919052 & 2.656971 \\ \mathrm{C} & -0.646935 & 1.147532 & 2.138596 \\ \mathrm{H} & -0.108481 & 2.076738 & 2.021887 \\ \mathrm{H} & -1.718402 & 1.186057 & 2.015052 \\ \mathrm{P} & -2.397166 & -1.001274 & 0.222792 \\ \mathrm{~N} & -2.938675 & -0.308222 & -1.233024 \\ \mathrm{C} & -3.343322 & 1.089326 & -1.156740 \\ \mathrm{H} & -2.480099 & 1.762821 & -1.227661 \\ \mathrm{H} & -4.028831 & 1.316409 & -1.977929 \\ \mathrm{H} & -3.859325 & 1.285073 & -0.218756 \\ \mathrm{C} & -2.295589 & -0.624270 & -2.501883 \\ \mathrm{H} & -1.508034 & 0.095174 & -2.745734 \\ \mathrm{H} & -1.850651 & -1.613633 & -2.459819 \\ \mathrm{H} & -3.045013 & -0.603946 & -3.301223 \\ \mathrm{~N} & -2.102821 & -2.594836 & -0.270300\end{array}$

$\begin{array}{lrrr}\mathrm{C} & -3.181177 & -3.412145 & -0.796112 \\ \mathrm{H} & -3.873769 & -2.802134 & -1.371656 \\ \mathrm{H} & -2.768613 & -4.177466 & -1.461079 \\ \mathrm{H} & -3.744348 & -3.922933 & -0.003088 \\ \mathrm{C} & -1.046089 & -3.348196 & 0.379723 \\ \mathrm{H} & -0.251554 & -2.671441 & 0.685631 \\ \mathrm{H} & -1.408356 & -3.906079 & 1.254920 \\ \mathrm{H} & -0.622569 & -4.070250 & -0.325123 \\ \mathrm{~N} & -3.895714 & -0.943583 & 1.065475 \\ \mathrm{C} & -3.868713 & -1.069481 & 2.508033 \\ \mathrm{H} & -2.853388 & -0.930985 & 2.879537 \\ \mathrm{H} & -4.507955 & -0.313203 & 2.979163 \\ \mathrm{H} & -4.219370 & -2.055324 & 2.842183 \\ \mathrm{C} & -5.227451 & -1.079970 & 0.499921 \\ \mathrm{H} & -5.673279 & -2.052306 & 0.748087 \\ \mathrm{H} & -5.891842 & -0.300172 & 0.891367 \\ \mathrm{H} & -5.181916 & -0.983525 & -0.581143\end{array}$

21d:

$$
\begin{aligned}
& E=-1141.72444761219 \mathrm{au} \\
& \mathrm{ZPE}=0.25241508 \mathrm{au} \\
& \mathrm{G}_{\text {corr }}=0.21273889 \mathrm{au} \\
& \mathrm{H}_{\text {corr }}=0.26825395 \mathrm{au}
\end{aligned}
$$




$\begin{array}{lrrr}\mathrm{S} & -1.304709 & -0.508195 & 0.398654 \\ \mathrm{P} & -1.862415 & -1.428863 & -1.222545 \\ \mathrm{~N} & -1.981813 & -0.495645 & -2.622599 \\ \mathrm{C} & -3.059251 & 0.493487 & -2.649621 \\ \mathrm{H} & -2.827611 & 1.362083 & -2.021760 \\ \mathrm{H} & -3.198988 & 0.832991 & -3.678002 \\ \mathrm{H} & -3.987145 & 0.053063 & -2.296021 \\ \mathrm{C} & -0.728684 & 0.070413 & -3.124330 \\ \mathrm{H} & -0.403904 & 0.927017 & -2.522243 \\ \mathrm{H} & 0.059060 & -0.677468 & -3.106579 \\ \mathrm{H} & -0.878521 & 0.402061 & -4.153917 \\ \mathrm{~N} & -0.757581 & -2.593153 & -1.711750 \\ \mathrm{C} & -0.875074 & -3.292843 & -2.982824 \\ \mathrm{H} & -1.417711 & -2.688050 & -3.703621 \\ \mathrm{H} & 0.124909 & -3.486976 & -3.382353\end{array}$

$\begin{array}{lrrr}\mathrm{H} & -1.385177 & -4.258264 & -2.875760 \\ \mathrm{C} & 0.044713 & -3.312708 & -0.734050 \\ \mathrm{H} & 0.132285 & -2.725292 & 0.175460 \\ \mathrm{H} & -0.395019 & -4.288044 & -0.488862 \\ \mathrm{H} & 1.045436 & -3.484406 & -1.141406 \\ \mathrm{~N} & -3.413920 & -2.049812 & -1.100715 \\ \mathrm{C} & -4.047447 & -2.314628 & 0.180678 \\ \mathrm{H} & -3.549736 & -1.747255 & 0.961509 \\ \mathrm{H} & -5.098838 & -2.013268 & 0.136912 \\ \mathrm{H} & -4.009664 & -3.380962 & 0.436496 \\ \mathrm{C} & -4.047902 & -2.733595 & -2.217467 \\ \mathrm{H} & -3.950339 & -3.823618 & -2.141114 \\ \mathrm{H} & -5.115662 & -2.494620 & -2.232632 \\ \mathrm{H} & -3.616926 & -2.408549 & -3.160465\end{array}$

20e:

$\mathrm{E}=-640.238654083942 \mathrm{au}$

$\mathrm{ZPE}=0.00842149 \mathrm{au}$

$\mathrm{G}_{\text {corr }}=-0.01769521 \mathrm{au}$

$\mathrm{H}_{\text {corr }}=0.01339352 \mathrm{au}$

$\begin{array}{llll}\text { P } & -0.379686 & -1.853730 & -1.007807 \\ \mathrm{~F} & -1.743681 & -1.676924 & -0.228561\end{array}$

F $\quad-0.677938$

$-0.844842-2.188130$

F $\quad-0.748628$

$-3.186227$

$-1.775533$

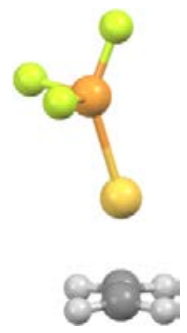

TS-i(11+20e $\rightarrow$ 4+21e): $\quad \quad E=-1116.36703349354 \mathrm{au}$

$$
\begin{aligned}
& Z P E=0.06285875 \mathrm{au} \\
& G_{\text {corr }}=0.02953662 \mathrm{au} \\
& v=-429.88 \mathrm{~cm}^{-1}
\end{aligned}
$$

$\begin{array}{lrrr}\mathrm{S} & -0.059108 & 0.039410 & -0.032815 \\ \mathrm{C} & 0.059321 & 0.036506 & 2.188564 \\ \mathrm{H} & 1.117683 & 0.122381 & 2.387747 \\ \mathrm{H} & -0.416884 & -0.892881 & 2.465138 \\ \mathrm{C} & -0.682294 & 1.132449 & 1.814065 \\ \mathrm{H} & -0.225030 & 2.104648 & 1.701747\end{array}$

$\begin{array}{lrrr}\mathrm{H} & -1.761141 & 1.089812 & 1.774013 \\ \mathrm{P} & 0.841760 & -0.315394 & -2.169362 \\ \mathrm{~F} & 0.492068 & -0.891189 & -3.606407 \\ \mathrm{~F} & 1.455880 & 1.048724 & -2.652314 \\ \mathrm{~F} & 2.171183 & -1.122959 & -1.948292\end{array}$

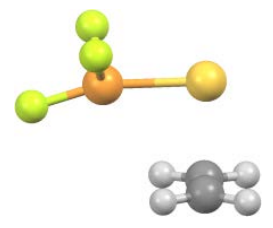

TS-ii (11+20e $\rightarrow$ 4+21e): $\quad E=-1116.3053556726$ au 


$$
\begin{aligned}
& Z P E=0.06264984 \mathrm{au} \\
& G_{\text {corr }}=0.02914374 \mathrm{au} \\
& v=-518.38 \mathrm{~cm}^{-1}
\end{aligned}
$$

$\begin{array}{lrrr}\mathrm{S} & 0.015799 & -0.017467 & 0.002101 \\ \mathrm{C} & 0.004555 & 0.002021 & 2.487212 \\ \mathrm{H} & 1.069043 & 0.019126 & 2.673638 \\ \mathrm{H} & -0.510091 & -0.925880 & 2.689430 \\ \mathrm{C} & -0.666499 & 1.118494 & 2.096717 \\ \mathrm{H} & -0.162416 & 2.065032 & 1.962812\end{array}$

$\begin{array}{lrrr}\mathrm{H} & -1.739761 & 1.118115 & 1.974776 \\ \mathrm{P} & -1.952238 & -1.119586 & 0.076902 \\ \mathrm{~F} & -3.367954 & -1.769801 & 0.553883 \\ \mathrm{~F} & -2.576350 & -0.424596 & -1.190418 \\ \mathrm{~F} & -1.420925 & -2.456105 & -0.562603\end{array}$

21e:

$$
\begin{aligned}
& \mathrm{E}=-1038.00138662271 \mathrm{au} \\
& \mathrm{ZPE}=0.01197086 \mathrm{au} \\
& \mathrm{G}_{\text {corr }}=-0.01613925 \mathrm{au} \\
& \mathrm{H}_{\text {corr }}=0.01784747 \mathrm{au}
\end{aligned}
$$

$\begin{array}{llll}\mathrm{S} & -0.794256 & -0.199252 & -0.704979 \\ \mathrm{P} & -1.641301 & -1.678977 & -1.483005 \\ \mathrm{~F} & -3.190511 & -1.728144 & -1.467865\end{array}$

20f:

$$
\begin{aligned}
& E=-1720.14911011365 \mathrm{au} \\
& \mathrm{ZPE}=0.00470122 \mathrm{au} \\
& \mathrm{G}_{\text {corr }}=-0.02475261 \mathrm{au} \\
& \mathrm{H}_{\text {corr }}=0.01085716 \mathrm{au}
\end{aligned}
$$

$\begin{array}{lrrr}\mathrm{P} & 0.000000 & 0.001242 & 0.732937 \\ \mathrm{Cl} & -0.000000 & 1.840782 & -0.215250\end{array}$

Cl 1.591636

Cl -1.591636

$-0.921012$

$-0.921012$

$-0.215898$

$-0.215898$
TS-i(11+20f $\rightarrow$ 4+21f):

$\begin{array}{rrrr}\mathrm{S} & -0.021944 & 0.024937 & -0.011915 \\ \mathrm{C} & 0.038263 & 0.019558 & 2.201162 \\ \mathrm{H} & 1.092287 & 0.077199 & 2.430651 \\ \mathrm{H} & -0.471961 & -0.894085 & 2.469328 \\ \mathrm{C} & -0.663845 & 1.135850 & 1.801526 \\ \mathrm{H} & -0.176696 & 2.094826 & 1.702252\end{array}$

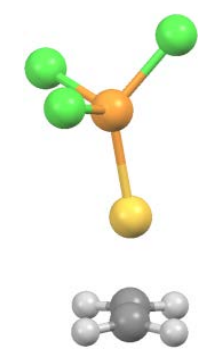

$\mathrm{E}=-2196.28090394697 \mathrm{au}$

$\mathrm{ZPE}=0.05866447 \mathrm{au}$

$\mathrm{G}_{\text {corr }}=0.02375925 \mathrm{au}$

$v=-430.04 \mathrm{~cm}^{-1}$ 
TS-ii $(\mathbf{1 1}+\mathbf{2 0 f} \rightarrow \mathbf{4 + 2 1 f}): \quad \mathrm{E}=-2196.22641050682 \mathrm{au}$

$$
\begin{aligned}
& \mathrm{ZPE}=0.058399725 \mathrm{au} \\
& \mathrm{G}_{\text {corr }}=0.02303866 \mathrm{au} \\
& v=-478.79 \mathrm{~cm}^{-1}
\end{aligned}
$$

$\begin{array}{lrrrrrrr}\mathrm{S} & 0.003674 & 0.004638 & -0.002852 & \mathrm{H} & -1.738621 & 1.147379 & 1.924951 \\ \mathrm{C} & 0.001798 & -0.002393 & 2.401916 & \mathrm{P} & -1.899430 & -1.080138 & 0.050341 \\ \mathrm{H} & 1.065457 & 0.003716 & 2.593055 & \mathrm{Cl} & -3.822043 & -1.831529 & 1.056949 \\ \mathrm{H} & -0.531164 & -0.914627 & 2.631367 & \mathrm{Cl} & -2.779533 & -0.223499 & -1.592276 \\ \mathrm{C} & -0.661279 & 1.130294 & 2.013934 & \mathrm{Cl} & -1.244049 & -2.885115 & -0.669642 \\ \mathrm{H} & -0.144796 & 2.070894 & 1.885880 & & & & \end{array}$

21f:

$E=-2117.90128288417$ au

$\mathrm{ZPE}=0.00744737 \mathrm{au}$

\begin{tabular}{|c|c|c|c|c|c|c|}
\hline 00 & 428 & 0.374892 & C & -1.577996 & -0.912999 & -0.530012 \\
\hline-0.0 & 1.823940 & 3111 & $\mathrm{~S}$ & -0.000001 & 0.001633 & 9190 \\
\hline
\end{tabular}

$\mathrm{G}_{\text {corr }}=-0.02373453 \mathrm{au}$

$\mathrm{H}_{\text {corr }}=0.01475860 \mathrm{au}$

20g $\left(\mathrm{PBr}_{3}\right)$ :

$\begin{array}{llll}\text { P } & 0.005894 & 0.009098 & 0.011985\end{array}$

$\begin{array}{llll}\text { Br } & 2.256124 & -0.007761 & -0.011358\end{array}$

$$
\begin{aligned}
& E=-8058.76616289788 \mathrm{au} \\
& Z P E=0.00342652 \mathrm{au} \\
& G_{\text {corr }}=-0.02950185 \mathrm{au} \\
& H_{\text {corr }}=0.01027268 \mathrm{au}
\end{aligned}
$$

21g $\left(\mathrm{S}=\mathrm{PBr}_{3}\right)$ :

S6:

$$
\begin{aligned}
& E=-8456.51354074831 \mathrm{au} \\
& Z P E=0.00584872 \mathrm{au} \\
& G_{\text {corr }}=-0.02873389 \mathrm{au} \\
& H_{\text {corr }}=0.01404029 \mathrm{au}
\end{aligned}
$$

$\begin{array}{rrrr}\mathrm{Br} & -0.426980 & 2.217401 & -0.014303 \\ \mathrm{Br} & -0.428432 & -0.513116 & 2.158126\end{array}$

$\begin{array}{lrrrrrrr}\mathrm{P} & -0.013875 & -0.000718 & -0.000006 & \mathrm{Br} & -0.984864 & -0.994949 & -1.734985 \\ \mathrm{~S} & 1.884663 & -0.007558 & 0.000012 & \mathrm{Br} & -0.984851 & -0.994934 & 1.734976 \\ \mathrm{Br} & -0.992445 & 1.997025 & 0.000005 & & & & \end{array}$




$$
\begin{aligned}
& \mathrm{ZPE}=0.00896637 \mathrm{au} \\
& \mathrm{G}_{\text {corr }}=-0.02353132 \mathrm{au} \\
& \mathrm{H}_{\text {corr }}=0.01762471 \mathrm{au}
\end{aligned}
$$

$\begin{array}{rrrrrrrr}\mathrm{S} & 0.077734 & -0.084083 & -0.024110 & \mathrm{~S} & 0.903625 & 1.733579 & -0.608343 \\ \mathrm{~S} & -0.034240 & 0.109197 & 2.044350 & \mathrm{~S} & 2.778978 & 1.733405 & 2.044111 \\ \mathrm{~S} & 1.953086 & -0.084257 & 2.628342 & \mathrm{~S} & 2.890951 & 1.540128 & -0.024350\end{array}$

$$
\mathrm{S}_{7}:
$$

S8:

$\begin{array}{lrrr}\text { S } & -0.240160 & -0.326738 & 0.049450 \\ \text { S } & 0.101558 & 0.351252 & 2.129031 \\ \text { S } & 2.009515 & -0.015840 & 2.589144 \\ \text { S } & 2.109048 & -2.056419 & 3.157582\end{array}$

$\mathrm{E}=-2784.15787565032 \mathrm{au}$

$\mathrm{ZPE}=0.01044737 \mathrm{au}$

$\mathrm{G}_{\text {corr }}=-0.02499324 \mathrm{au}$

$\mathrm{H}_{\text {corr }}=0.02088017 \mathrm{au}$

\section{CS:}

C

$\begin{array}{lrrr}\text { S } & 0.073105 & 0.033116 & -0.021829 \\ \text { S } & -0.033646 & -0.072498 & 2.041706 \\ \text { S } & 1.891329 & 0.032450 & 2.792261 \\ \text { S } & 2.527683 & -1.891545 & 3.207212\end{array}$

$$
\begin{aligned}
& E=-3181.90601776822 \mathrm{au} \\
& \mathrm{ZPE}=0.01212175 \mathrm{au} \\
& \mathrm{G}_{\text {corr }}=-0.02554497 \mathrm{au} \\
& \mathrm{H}_{\text {corr }}=0.02411310 \mathrm{au}
\end{aligned}
$$

$\begin{array}{llll}\mathrm{S} & 3.031443 & -2.927880 & 1.533265\end{array}$

$\begin{array}{llll}\mathrm{S} & 1.605307 & -3.058925 & 0.051945\end{array}$

$\begin{array}{llll}\mathrm{S} & 1.480283 & -1.060886 & -0.648067\end{array}$

$\begin{array}{lrrr}\text { S } & 3.689870 & -2.540112 & 1.623481 \\ \text { S } & 2.507672 & -3.712288 & 0.396359 \\ \text { S } & 1.868782 & -2.544687 & -1.187293 \\ \text { S } & -0.049323 & -1.891105 & -0.771897\end{array}$
$\mathrm{CS}_{2}$ :
$E=-833.497223838675 \mathrm{au}$
$\mathrm{ZPE}=0.00706579 \mathrm{au}$
$\mathrm{G}_{\text {corr }}=-0.01583836 \mathrm{au}$
$\mathrm{H}_{\text {corr }}=0.01107871 \mathrm{au}$
$\begin{array}{llll}\text { C } & 0.000000 & 0.000000 & -0.000000\end{array}$
$\begin{array}{llll}\text { S } & -0.000000 & -0.000000 & -1.553047\end{array}$
$\begin{array}{llll}\text { S } & -0.000000 & -0.000000 & 1.553047\end{array}$ 
$\mathrm{CO}:$

$$
E=-113.157694712963 \mathrm{au}
$$

$$
\mathrm{ZPE}=0.00504032 \mathrm{au}
$$$$
\mathrm{G}_{\text {corr }}=-0.01342344 \mathrm{au}
$$$$
\mathrm{H}_{\text {corr }}=0.00834522 \mathrm{au}
$$
C
0.251432
$-4.298698 \quad-13.159716$
O $\quad 0.567740$
$-3.510812 \quad-13.897941$

$\begin{array}{lrrr}\mathrm{O}=\mathrm{C}=\mathrm{S}: & & \mathrm{E}= \\ & & \mathrm{ZPE} \\ & & \mathrm{G}_{\mathrm{cor}} \\ & & \\ & & \\ & & & \\ & & \\ \mathrm{C} & 0.404511 & -1.218611 & -2.093443 \\ \mathrm{O} & -0.487684 & -0.677708 & -2.590282\end{array}$

Me-NC:

$$
\begin{aligned}
& \mathrm{E}=-132.490729795161 \mathrm{au} \\
& \mathrm{ZPE}=0.04503616 \mathrm{au} \\
& \mathrm{G}_{\text {corr }}=0.02189183 \mathrm{au} \\
& \mathrm{H}_{\text {corr }}=0.04981674 \mathrm{au}
\end{aligned}
$$

$\begin{array}{lrrr}\mathrm{C} & 0.865574 & 0.969312 & 0.017290 \\ \mathrm{~N} & -0.284288 & 0.784181 & 0.011442 \\ \mathrm{C} & -1.684557 & 0.557969 & 0.003881\end{array}$

H $\quad-2.130441$

$\mathrm{H} \quad-2.130813$

H $\quad-1.885137$

0.973756

1.036225

0.907341

$-0.867953$

$-0.512664 \quad-0.033630$
Me-NCS:

Et-NC:

$\begin{array}{lrrr}\mathrm{S} & 1.819566 & 2.556288 & 0.073436 \\ \mathrm{C} & 0.555631 & 1.605428 & 0.039883 \\ \mathrm{~N} & -0.341636 & 0.833563 & 0.012507 \\ \mathrm{C} & -1.731824 & 0.528278 & 0.003005\end{array}$

$E=-530.254438130 \mathrm{au}$

$\mathrm{ZPE}=0.04785549 \mathrm{au}$

$G_{\text {corr }}=0.02183903 \mathrm{au}$

$\mathrm{H}_{\text {corr }}=0.05304505 \mathrm{au}$

$$
\begin{aligned}
& E=-171.733039416712 \mathrm{au} \\
& Z P E=0.07381195 \mathrm{au} \\
& \mathrm{G}_{\text {corr }}=0.04804198 \mathrm{au} \\
& \mathrm{H}_{\text {corr }}=0.07961414 \mathrm{au}
\end{aligned}
$$

$\begin{array}{lrrrrrrr}\mathrm{C} & 0.864138 & 0.977462 & 0.017701 & \mathrm{C} & -1.987288 & -0.958326 & -0.049353 \\ \mathrm{~N} & -0.286427 & 0.794420 & 0.011714 & \mathrm{H} & -3.066775 & -1.115042 & -0.053426 \\ \mathrm{C} & -1.688221 & 0.536240 & 0.003103 & \mathrm{H} & -1.566606 & -1.403997 & -0.950702 \\ \mathrm{H} & -2.118123 & 0.986241 & 0.900120 & \mathrm{H} & -1.564112 & -1.466470 & 0.817073 \\ \mathrm{H} & -2.118714 & 1.048091 & -0.859818 & & & & \end{array}$


Et-NCS:

Phosphirane:

Phosphirane P-sulfide:

$$
\begin{aligned}
& E=-569.497101360752 \mathrm{au} \\
& \mathrm{ZPE}=0.07651552 \mathrm{au} \\
& \mathrm{G}_{\text {corr }}=0.04839812 \mathrm{au} \\
& \mathrm{H}_{\text {corr }}=0.08273254 \mathrm{au}
\end{aligned}
$$

$\begin{array}{lrrr}\mathrm{S} & 1.799456 & 2.575530 & 0.072475 \\ \mathrm{C} & 0.552572 & 1.602444 & 0.040090 \\ \mathrm{~N} & -0.332655 & 0.815475 & 0.014077 \\ \mathrm{C} & -1.732002 & 0.506238 & 0.002391 \\ \mathrm{H} & -2.187100 & 0.935139 & 0.899911\end{array}$

$\begin{array}{lrrr}\mathrm{H} & -2.186333 & 0.997622 & -0.862912 \\ \mathrm{C} & -1.976661 & -0.995109 & -0.050836 \\ \mathrm{H} & -3.049278 & -1.194704 & -0.056210 \\ \mathrm{H} & -1.539086 & -1.426018 & -0.951790 \\ \mathrm{H} & -1.536109 & -1.488990 & 0.815655\end{array}$

\begin{tabular}{lrrr}
\multicolumn{3}{c}{ Phosphirane: } & \multicolumn{2}{c}{ E } \\
& & & $\mathrm{ZP}$ \\
& & $\mathrm{G}_{\mathrm{c}}$ \\
& & & $\mathrm{H}_{\mathrm{c}}$ \\
& & & \\
& & & \\
$\mathrm{P}$ & -0.095041 & -0.027826 & 0.114475 \\
$\mathrm{H}$ & -0.686074 & 1.185468 & 0.558331 \\
$\mathrm{C}$ & -1.547360 & -1.113601 & 0.559637 \\
$\mathrm{H}$ & -2.504206 & -0.633163 & 0.711308
\end{tabular}

$E=-419.944460583298 \mathrm{au}$

$\mathrm{ZPE}=0.06308983 \mathrm{au}$

$\mathrm{G}_{\text {corr }}=0.03864783 \mathrm{au}$

$\mathrm{H}_{\text {corr }}=0.06769002 \mathrm{au}$

H $\quad-1.605410$

$-2.043405$

0.007555

C -0.525385

$-1.012108$

1.641434

$\begin{array}{llll}\mathrm{H} & 0.106760 & -1.873383 & 1.818539\end{array}$

$\begin{array}{llll}\mathrm{H} & -0.779363 & -0.462540 & 2.537469\end{array}$

H-P-Mo(CO)5:

$$
\begin{aligned}
& E=-975.194612278 \mathrm{au} \\
& Z P E=0.04848606 \mathrm{au} \\
& \mathrm{G}_{\text {corr }}=0.00588394 \mathrm{au} \\
& \mathrm{H}_{\text {corr }}=0.06379109 \mathrm{au}
\end{aligned}
$$

$\begin{array}{lrrr}\mathrm{P} & -0.081772 & -2.133584 & 1.047695 \\ \mathrm{H} & 1.209153 & -2.749734 & 1.014501 \\ \mathrm{Mo} & 0.087942 & -0.519766 & 2.802982 \\ \mathrm{C} & 0.180969 & 0.932104 & 4.317934 \\ \mathrm{O} & 0.241740 & 1.718644 & 5.138079 \\ \mathrm{C} & 1.322799 & 0.696880 & 1.646196 \\ \mathrm{O} & 1.998838 & 1.362205 & 1.019408\end{array}$

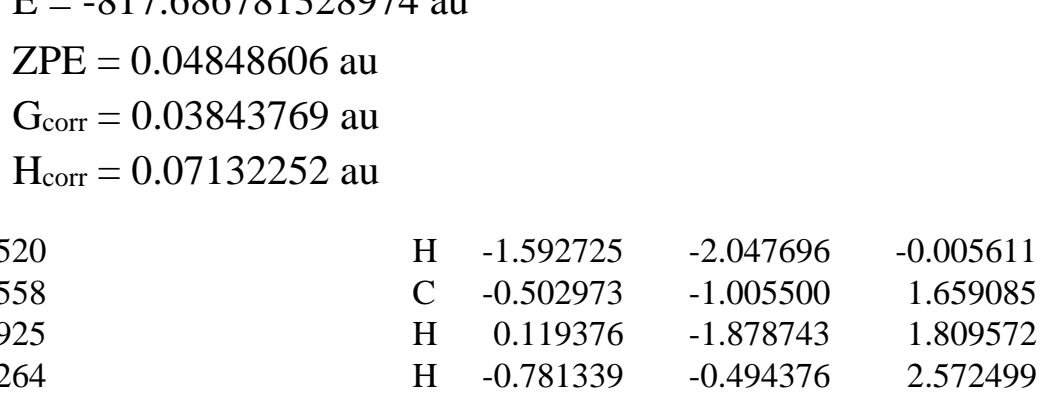

$\begin{array}{lrrr}\mathrm{P} & -0.167325 & -0.036157 & 0.181520 \\ \mathrm{H} & -0.607465 & 1.263689 & 0.479558 \\ \mathrm{~S} & 1.229518 & -0.288914 & -1.128925 \\ \mathrm{C} & -1.565100 & -1.110544 & 0.536264 \\ \mathrm{H} & -2.538527 & -0.671230 & 0.715863\end{array}$

(1)

$\begin{array}{lrrr}\mathrm{C} & -1.594385 & 0.328220 & 1.968547 \\ \mathrm{O} & -2.520570 & 0.780327 & 1.481930 \\ \mathrm{C} & -1.139707 & -1.661667 & 4.050603 \\ \mathrm{O} & -1.814233 & -2.278622 & 4.726026 \\ \mathrm{C} & 1.766792 & -1.531537 & 3.426446 \\ \mathrm{O} & 2.696253 & -2.124438 & 3.715579\end{array}$


Styrene:

$$
\begin{aligned}
& E=-309.05854176788 \mathrm{au} \\
& Z P E=0.13294853 \mathrm{au} \\
& G_{\text {corr }}=0.10242017 \mathrm{au} \\
& H_{\text {corr }}=0.14068646 \mathrm{au}
\end{aligned}
$$

$\begin{array}{rrrr}\mathrm{C} & -0.031192 & 0.000292 & 0.055489 \\ \mathrm{H} & -0.163539 & 0.000407 & 1.128955 \\ \mathrm{H} & 0.989991 & -0.000736 & -0.304469 \\ \mathrm{C} & -1.082407 & 0.001373 & -0.763187 \\ \mathrm{H} & -2.074754 & 0.002372 & -0.321056 \\ \mathrm{C} & -1.070198 & 0.001394 & -2.230209 \\ \mathrm{C} & -2.288650 & 0.002692 & -2.917341 \\ \mathrm{C} & -2.333030 & 0.002787 & -4.305510\end{array}$

$\begin{array}{rrrr}\mathrm{C} & -1.153546 & 0.001584 & -5.038511 \\ \mathrm{C} & 0.068409 & 0.000275 & -4.369742 \\ \mathrm{C} & 0.110031 & 0.000176 & -2.985148 \\ \mathrm{H} & -3.212144 & 0.003630 & -2.350363 \\ \mathrm{H} & -3.289132 & 0.003802 & -4.813435 \\ \mathrm{H} & -1.182273 & 0.001657 & -6.120533 \\ \mathrm{H} & 0.992886 & -0.000675 & -4.933459 \\ \mathrm{H} & 1.069508 & -0.000865 & -2.485146\end{array}$

Phenylthiirane:

$$
\begin{aligned}
& E=-706.794928393446 \mathrm{au} \\
& \mathrm{ZPE}=0.13599830 \mathrm{au} \\
& \mathrm{G}_{\text {corr }}=0.10383827 \mathrm{au} \\
& \mathrm{H}_{\text {corr }}=0.14456043 \mathrm{au}
\end{aligned}
$$

$\begin{array}{lrrrrrrr}\mathrm{S} & -0.227886 & -0.887462 & 0.891966 & \mathrm{C} & -4.700344 & 2.224495 & 1.482714 \\ \mathrm{C} & 0.180756 & 0.444088 & 2.058354 & \mathrm{C} & -4.235207 & 1.114798 & 2.183958 \\ \mathrm{H} & 1.220207 & 0.748126 & 2.076210 & \mathrm{C} & -2.935583 & 0.666591 & 2.003789 \\ \mathrm{H} & -0.289093 & 0.373610 & 3.031785 & \mathrm{H} & -1.894816 & 2.943192 & -0.274625 \\ \mathrm{C} & -0.662993 & 0.903925 & 0.928285 & \mathrm{H} & -4.208293 & 3.737842 & 0.040585 \\ \mathrm{H} & -0.144575 & 1.464107 & 0.159028 & \mathrm{H} & -5.716831 & 2.569261 & 1.622380 \\ \mathrm{C} & -2.074803 & 1.324194 & 1.122107 & \mathrm{H} & -4.892122 & 0.592834 & 2.868254 \\ \mathrm{C} & -2.551270 & 2.429375 & 0.417788 & \mathrm{H} & -2.590497 & -0.217013 & 2.525549 \\ \mathrm{C} & -3.855080 & 2.878673 & 0.596564 & & & & \end{array}$

